\title{
Especialización tecnológica, captura y formación de competencias bajo integración de mercados: comparación entre Asia y América Latina
}

\author{
Ana Urraca Ruiz, ${ }^{* *}$
}

\begin{abstract}
Resumen
Este trabajo tiene como objetivo examinar la evolución de la especialización, convergencia y el proceso de formación de competencias tecnológicas de dos conjuntos de países - latinoamericanos y asiáticos - entre los periodos 1985-1995 y 1999-2008. Entre ambos periodos, la mayor parte de estos países registraron mayores niveles de integración con el mundo en sus mercados de bienes, servicios y capitales. El trabajo concluye: i) no existe un único patrón de convergencia; ii) el grupo latinoamericano responde a un patrón de especialización sobre tecnologías vinculadas a recursos naturales mientras que el asiático explota el paradigma electrónico; iii) la persistencia es un fenómeno ligado a la des-especialización y se concentra en competencias centrales y marginales; iv) ante elevados ritmos de captura, la movilidad puede constituirse más relevante que la persistencia y es escasamente explicada por la acumulación tecnológica; v) no hay evidencia de que la especialización latinoamericana en tecnologías de oportunidad tecnológica estancada haya supuesto una restricción a su dinamismo tecnológico.
\end{abstract}

Palabras clave: Especialización tecnológica; Captura; Cambio estructural; Asia; América Latina.

\section{Abstract \\ Technological specialization, catching-up and building of technological competences under market integration: Asia and Latin America compared}

The aim of this paper is to observe the evolution of technological specialization, convergence and the process of building technological competences for two groups of countries - Asia and Latin America - between 1985-1995 and 1999-2008. Between these periods, most of the countries in these groups registered growing levels of global integration in terms of goods, services and capital markets. The main conclusions of the study are: i) there is no unique pattern of technological convergence between countries; ii) Latin-American countries specialize in technologies linked to natural resources while the Asian countries explored the electronic and telecommunication paradigms; iii) persistency is a phenomenon associated to de-specialization and is concentrated in marginal and core competences; iv) considering rapid rates of catch-up, mobility can become more relevant than persistency and can be only marginally explained by technological accumulation; v) there is no evidence that LatinAmerican specialization on stagnant technologies would be a constraint to its technological dynamism.

Keywords: Technological specialization; Catching-up; Structural change; Asia; Latin America. JEL O3, O5.

\footnotetext{
* Trabalho recebido em 15 de janeiro de 2011 e aprovado em 21 de maio de 2012.

** Coordinadora del Programa de posgrado de Economía de la Facultad de Economía de la Universidad Federal Fluminense, Niterói, RJ, Brasil. E-mail: anaruiz@economia.uff.br.
} 


\section{Introducción}

La década de los noventa representó un punto de inflexión en el fenómeno económico mundial conocido como "globalización" que supuso un mayor grado de integración entre los países y que afectó a diversas dimensiones de las economías. En el ámbito tecnológico, los países comenzaron a cambiar su patrón de especialización tecnológica nacional (ETN). En la transición de la década de los ochenta a los noventa, los países industrializados habían mostrado una tendencia hacia la especialización de sus perfiles tecnológicos (Archibugi; Pianta, 1992, 1994; Mancusi, 2001), mientras que los países en desarrollo tendían a concentrar su actividad innovadora en áreas tecnológicamente estancadas en el nivel mundial (Montobbio; Rampa, 2005).

Una de las principales características asociadas a la ETN es su persistencia a lo largo del tiempo. De acuerdo con esta idea, la evidencia empírica debería mostrar la dificultad de los países en crear especializaciones en campos técnicos donde no existen esfuerzos previos ni capacidad de absorción y una facilidad en aumentar la especialización en los campos técnicos donde el país se especializó en el pasado (y en sus campos técnicos relacionados). La idea de persistencia conduce a 'irreversibilidad', esto es, la imposibilidad de que los patrones de especialización se dirijan hacia campos técnicos donde no hay especialización previa y la constante permanencia en especializaciones previas. Los trabajos empíricos más recientes constataron la importancia de la persistencia en los patrones de especialización cuando existían altas des-especializaciones, siendo éste un fenómeno más asociado a países líderes (Mancusi, 2001). Sin embargo, la movilidad entre clases tecnológicas podía ser elevada y asimétrica para determinados grados de desagregación $^{1}$ (Stolpe, 1995; Mancusi, 2001). La asimetría consistía en la dificultad de mejorar la especialización en muchas tecnologías donde existía desventaja (y en sus áreas vinculadas), y una relativa facilidad de diversificación y cambio de especialización en las áreas técnicas donde existían elevadas ventajas comparativas (y en sus vinculadas). Las explicaciones teóricas para estos encuentros permanecen todavía poco claras.

Tres observaciones deben ser realizadas sobre la literatura acerca de la ETN. Primero, desde un punto de vista teórico fueron desconsiderados los efectos de la integración de mercados sobre la asignación de recursos a la innovación, aunque la mayor parte de los estudios empíricos fue realizada para países que estaban pasando por procesos de transición hacia espacios integrados. En segundo lugar, la mayor parte de los trabajos empíricos fue realizada sobre países industrializados y no hay apenas evidencia empírica sobre cuál fue la evolución de

(1) El nivel de desagregación está relacionado con la persistencia de la estructura tecnológica. Cuanto mayor el grado de agregación, mayor será también la persistencia y cuanto mayor la desagregación, mayor la movilidad (Malerba; Montobbio, 2003). 
los patrones de especialización de países en desarrollo, sobre cómo la integración de mercados en estos países pudo haber afectado al proceso de adopción, absorción y generación de tecnologías (captura tecnológica), ni sobre cómo este proceso de captura ha podido alterar las distancias tecnológicas de estos países con el mundo. Si la captura tiene lugar en tecnologías que representan especializaciones previas, el resultado del proceso será un aumento de la especialización. Si la captura afecta a la creación de competencias en nuevos campos de conocimiento (movilidad), el resultado del proceso será un aumento de diversificación de la base técnica. Ambos fenómenos no son excluyentes y pueden suceder simultáneamente, de forma que el resultado final del aumento de la especialización o de la diversificación de la base técnica dependerá de cuál de las dos tendencias es más fuerte.

En tercer lugar, existe una idea extendida en la literatura de que el paso hacia espacios integrados condujo a los países latinoamericanos y asiáticos a diferentes patrones de especialización de acuerdo con una nueva división internacional del trabajo. En cuanto los países emergentes asiáticos aprovecharon el impacto de la integración para especializarse en tecnologías permeables y dinámicas (electrónica y telecomunicaciones), los países latinoamericanos se especializaron en tecnologías estancadas y con pocos efectos expansivos (generalmente vinculadas a la explotación de recursos naturales), lo que supondría un límite en su crecimiento y en su futuro desarrollo tecnológico (Huang; Miozzo, 2004).

Bajo estas consideraciones, este trabajo tiene como objetivo comparar los principales cambios registrados en los patrones de especialización y convergencia tecnológica de un conjunto de países asiáticos y latinoamericanos entre los momentos anterior y posterior a su entrada en mercados más integrados, aunque sin el propósito de medir cómo la integración contribuyó a ello. El análisis se centra en la idea de que la captura tecnológica, en combinación con cambios en las estructuras productivas, puede inducir a la movilidad entre clases tecnológicas, lo que cuestiona la 'irreversibilidad' del cambio técnico a partir de 'incorrectas' especializaciones iniciales en países latinoamericanos.

Además de esta introducción, el trabajo se compone de tres partes. La primera trae los principales elementos teóricos y la evidencia empírica relativa a los determinantes de la evolución de la ETN haciendo un especial hincapié en cuáles serían los elementos relativos al cambio estructural que pueden tomar un papel relevante en la evolución de la ETN ante procesos de integración económica. La segunda cualifica el proceso de integración económica registrado en los países contemplados en el estudio a partir de indicadores sobre liberalización comercial y estructura de importaciones. La tercera parte realiza una evaluación del dinamismo tecnológico y de los cambios en el patrón de especialización tecnológica entre periodos pre y post integración. Esta parte también incluye un análisis del proceso 
de formación de competencias tecnológicas de estos países de forma que sea posible saber en qué medida las hipótesis sobre acumulación tecnológica consiguen explicar los cambios observados durante procesos de integración económica. El artículo finaliza con las principales conclusiones que pueden extraerse a este respecto.

\section{Integración económica y especialización tecnológica}

La ETN representa la regularidad de la actividad tecnológica de un país entre los diversos campos técnicos o de conocimiento, esto es, las áreas técnicas en las que el país desarrolla competencias o realiza emprendimientos tecnológicos de entre todas las posibilidades tecnológicas. La tradición neoshumpeteriana prevé que una determinada distribución de capacitaciones tecnológicas nacionales es el resultado de dos conjuntos de fuerzas; 1) de carácter autónomo, son las dirigidas por la propia dinámica de cambio técnico; y 2) de carácter inducido, representadas por los Sistemas Nacionales de Innovación y las políticas tecnológicas. A estos dos conjuntos de fuerzas hay que añadir un tercero, de carácter estructural, que surge de las relaciones entre las estructuras productiva, comercial y técnica (Urraca-Ruiz, 2013).

Las fuerzas autónomas del cambio técnico son los componentes de los llamados 'regímenes tecnológicos' los cuales permiten identificar los ritmos y trayectorias (paths) tecnológicos de acuerdo con las especificidades de cada tecnología en términos de apropiabilidad, oportunidad, acumulabilidad y demanda bajo rigurosas hipótesis sobre variedad y especificidad del conocimiento (Nelson y Winter, 1977; Dosi, 1988; Cohen, 1995). Entre todos ellos, la literatura ha centrado su atención en la oportunidad tecnológica y la acumulabilidad como determinantes de la ETN. La oportunidad tecnológica representa la posibilidad de explotar las oportunidades que surgen con la aparición de micro-paradigmas tecnológicos en el escenario internacional. Cuando esto sucede, puede tener lugar un reaprovechamiento de las competencias nacionales y una reasignación pública y privada de recursos a la innovación desde unas áreas tecnológicas hacia otras, lo que sumado al efecto "permeable" de las tecnologías, llevaría a una reconducción de la ETN y a la movilidad tecnológica (Mancusi, 2003). De esta forma, la oportunidad empuja a la movilidad, es decir, a cambios hacia una nueva distribución de la actividad tecnológica entre campos técnicos. Este efecto de la oportunidad tecnológica sobre la ETN ha conducido a la idea de que existen 'correctas' e 'incorrectas' especializaciones que determinan los perfiles futuros de especialización y dinamismo tecnológico. Las 'correctas' especializaciones son las realizadas sobre tecnologías con alto grado de permeabilidad o con elevado ritmo de crecimiento de su cuota de patentes, lo que significaría que son tecnologías que contaron con elevadas oportunidades tecnológicas a lo largo del tiempo (Huang; 
Miozzo, 2004; Meliciani 2002; Montobbio; Rampa, 2005). La especialización en este tipo de tecnologías concedería ciertas ventajas de dinamismo tecnológico al contar con un elevado potencial de aplicación de nuevo y genérico conocimiento científico y técnico en un mayor rango de actividades y al permitir el desarrollo de futuros procesos de aprendizaje (Huang; Miozzo, 2004). Paralelamente, la especialización en tecnologías 'inferiores' (o de baja oportunidad tecnológica) representaría una restricción a la movilidad hacia 'superiores' campos técnicos (de alta oportunidad), especialmente si no hay un apropiado marco institucional y políticas públicas que estimulen el 'proceso social de aprendizaje' (Vertova, 2001; Jungmittag, 2004). Sin embargo, la evidencia empírica muestra que, a pesar de contar con especializaciones iniciales desfavorables, los países retardatarios pueden registrar alto dinamismo tecnológico mediante la imitación y la captura, siempre que sean emprendidos esfuerzos en capacidad de absorción (Laursen, 1999; Meliciani, 2002).

La acumulación tecnológica representa la 'continuidad' del cambio técnico. El carácter idiosincrásico de los procesos de acumulación de conocimiento y las trayectorias tecnológicas de empresas y países lleva a la formación de ventajas particulares para ciertas tecnologías y de sus áreas técnicas vinculadas. Una vez formadas las ventajas, la naturaleza acumulativa de los procesos de aprendizaje, el carácter dominio-específico de la 'capacidad de absorción' (en sentido de Cohen; Levinthal, 1990) y la especificidad de las capacitaciones organizativas y tecnológicas de empresas y países llevarían a la persistencia de los patrones de especialización a lo largo del tiempo (Malerba et al., 1997; Brusoni; Geuda, 2003). En presencia de barreras a la difusión fuera de las fronteras nacionales, los spillovers del conocimiento adquieren un marcado carácter nacional, acentuado los fenómenos de path dependence y lock-in, es decir, el futuro reproduce el pasado y pequeñas diferencias iniciales entre países llevarían a la divergencia en los patrones de ETN y a la desigualdad en el crecimiento (Mancusi, 2001) en un elevado grado de irreversibilidad.

La demanda es también un importante factor que determina el ritmo y la dirección de las trayectorias tecnológicas nacionales porque el tamaño del mercado y la elasticidad de la demanda afectan al incentivo a innovar (Cohen, 1995, p. 214) y porque el mercado es un poderoso mecanismo de selección (Dosi, 1988). A pesar de su importancia, la demanda ha sido desconsiderada como factor explicativo en trabajos teóricos y empíricos sobre ETN. Su importancia adquiere un papel muy importante en condiciones de cambio del ambiente económico que generan un cambio estructural, como es la integración de mercados. La integración comercial puede conducir a la creación de nuevos mercados o a la expansión versus destrucción de los previos. Y esto puede suceder en la medida en que la integración altera la expectativa de crecimiento de unos mercados sobre otros. Estos cambios 
pueden llevar a una reasignación de los recursos a la innovación hacia tecnologías donde la rentabilidad esperada de los gastos en I+D es mayor. En espacios económicos integrados, los cambios en el ambiente económico y los mecanismos de selección de mercado y de 'no mercado' también registran alteraciones, concretamente, en los precios relativos de los insumos, en las necesidades y hábitos del consumidor, en los requerimientos regulatorios y en las formas en que se establecen los imperativos tecnológicos en el área integrada. El acceso a mercados integrados también altera los precios relativos de los recursos a la innovación, la adquisición de tecnologías incorporadas mediante importaciones de bienes se vuelve más barata y los flujos de capitales pueden facilitar la transferencia de tecnología de unos países a otros (Camerona et al., 2005). La captura tecnológica se acelera, especialmente en países retardatarios, pudiendo llegar incluso a desarrollar competencias en nuevas áreas técnicas. Este efecto llevaría a los países a diversificar su base técnica y los empujaría hacia la convergencia tecnológica con los países líderes, alterando definitivamente sus trayectorias tecnológicas sobre las que inicialmente seguirían bajo ambientes económicos restringidos a su propia realidad nacional.

Las fuerzas inducidas vienen representadas por los Sistemas Nacionales de Innovación (SNI). Los SNI contienen aspectos culturales, históricos y sociales que son específicos de cada país. La idiosincrasia de los SNI también responde a la especificidad de los procesos de innovación y de aprendizaje, lo cual refuerza el argumento de persistencia de la ETN y explica las diferencias de ETN entre países, así como los diferentes caminos que estos pueden seguir. La perspectiva de los SNI permite también identificar la acción del estado como un agente activo capaz de recomponer las competencias científicas y tecnológicas nacionales (Mancusi, 2001; Vertova, 2001; Brusoni; Geuna, 2003). El cambio de ambiente económico que representa la integración induce a estrategias que llevan consigo reasignación de los factores tecnológicos para acelerar el proceso de captura en países tecnológicamente retardatarios. La evidencia empírica ha mostrado que los estados no permanecen indiferentes ante procesos de integración económica, sino que estimulan la implementación de estrategias defensivas u ofensivas. Por ejemplo, la experiencia europea muestra que los Programas Plurianuales de I+D de la UE estimularon la implementación de políticas tecnológicas activas en todos los países miembros y estas políticas fueron especialmente importantes en países retardatarios, con SNI débiles y donde la actividad tecnológica de las empresas era reducida (España, Portugal, Irlanda o Grecia). Respuestas similares fueron observadas en algunos países asiáticos (China, India o Corea del Sur) donde los gobiernos tomaron un papel activo en la asignación de recursos tecnológicos para recomponer su base técnica desarrollando un papel fundamental en la creación de competencias tecnológicas en campos de conocimiento donde apenas existía capacitación nacional. 
El tercer conjunto de fuerzas que determina la evolución de la ETN es el relativo a los vínculos entre la estructura tecnológica y productiva. La estructura tecnológica viene determinada por tres elementos; la base técnica, los spillovers o interrelaciones (vínculos) entre tecnologías y las relaciones entre innovadores (Malerba; Montobbio, 2003). La base técnica viene dada por la dotación de científicos e ingenieros, por la asignación de recursos a la I+D por campo de conocimiento y por las diferentes formas en que el país adquiere y acumula conocimiento. La estructura productiva viene dada por la base productiva o patrón de especialización productiva (por ejemplo en industrias de alto, bajo o medio contenido tecnológico; intensivas en recursos naturales, conocimiento, factor capital o factor trabajo); por el grado de interdependencia o interrelación entre industrias, a lo largo de las cadenas productivas (tejido productivo); y por las relaciones de competencia entre empresas (concentración, poder de mercado, barreras a la entrada) o de cooperación y otras formas de coordinación.

Ambas estructuras están interconectadas. De un lado, las industrias cuentan con diferentes asignaciones de recursos a la $\mathrm{I}+\mathrm{D}$. Por tanto, los países que poseen una base técnica superior tienden a estar más especializados en industrias intensivas en conocimiento, mientras que países con inferior base técnica tienden a estar más especializados en industrias intensivas en factor trabajo (Stolpe, 1995, cap. D). Por otro lado, la base técnica está conectada con la base productiva en la medida en que estas definen bases específicas de conocimiento para cada industria relativas a las especificidades de los productos que generan y los procesos que utilizan. Sobre esas bases de conocimiento se construyen las competencias tecnológicas. Los spillovers entre tecnologías también están conectados con las interacciones entre industrias, dado que las cadenas productivas comparten alta complementariedad tecnológica. Finalmente, las interacciones entre innovadores están altamente relacionadas con las interacciones entre competidores a través de formas muy diversas: los acuerdos de colaboración, joint-ventures tecnológicas, ventajas del primer-movedor y poder de mercado, etc., son buenos ejemplos de ello.

A pesar de la existencia de este tipo de relaciones, la literatura sobre cambio estructural suele tratarlas separadamente. Sin embargo, el componente estructural puede tomar un papel protagonista para explicar la evolución de la ETN en ambientes que fuerzan importantes cambios en las estructuras productivas, como es el caso de la integración de mercados. Los vínculos entre ambas estructuras, pueden ayudar a entender la movilidad entre tecnologías que no puede ser explicada por los factores autónomos del cambio técnico ni por los factores inducidos. Algunos cambios esperados en la ETN derivados de cambios en la estructura productiva son los siguientes. Primero, las teorías del comercio internacional prevén que la integración comercial debería alterar la estructura productiva hacia una mayor especialización en aquellas actividades donde existen 
ventajas comparativas, generalmente vinculadas a ventajas en la dotación de factores. Como las competencias tecnológicas descansan en la base productiva, se espera que los países registren movilidad hacia las tecnologías que componen la base técnica de la nueva base productiva. Este tipo de movimientos puede ser especialmente importante en pequeñas economías que están de hecho altamente especializadas. Si este fenómeno sucede en todos los países y la especialización se da en los mismos campos técnicos, el efecto total será de mayor especialización con convergencia. Pero si la especialización de los países tiene lugar en diferentes campos técnicos, el efecto total será de divergencia (Archibugi; Pianta, 1994). Segundo, de acuerdo con Stolpe (1995, cap. D), la asignación de recursos a la I+D en economías abiertas puede responder a cambios en las dotaciones factoriales relativas a su patrón de comercio. Esto significaría un cambio del patrón de especialización hacia aquellas actividades en las que la dotación factorial relativa es una fuente de ventaja comparativa. Finalmente, la trayectoria de especialización tecnológica puede seguir la trayectoria de especialización productiva a lo largo del proceso de desarrollo tecnológico y del crecimiento. Desde un punto de vista empírico, se ha comprobado que la especialización productiva se mueve a lo largo del proceso de crecimiento económico siguiendo una curva en formato de U (Imbs; Wacziarg, 2003). Al principio, los países están fuertemente especializados en pocas actividades productivas, pero progresivamente van diversificando su estructura productiva a medida que avanza su proceso de industrialización. Cuando la industrialización madura, los países retornan a estructuras más especializadas. Una tendencia similar puede ser observada en la ETN cuando los procesos de captura, estimulados por la integración, aceleran el desarrollo tecnológico en países seguidores. Las fases iniciales del desarrollo tecnológico se corresponden con elevados grados de especialización en pocos campos técnicos y con alta desespecialización en muchos campos técnicos. Este escenario deberá cambiar a medida que los países alcancen mayores niveles de desarrollo tecnológico y una base productiva también más diversificada, de forma que puedan ser registrados mayores niveles de diversificación tecnológica. Pero una vez que las oportunidades tecnológicas maduran o se agotan, es posible un retorno a la concentración de la especialización, iniciando un nuevo ciclo en el patrón de especialización desde nuevas oportunidades tecnológicas.

\section{Evolución general comparada de la integración de mercados en América Latina y Asia (países seleccionados)}

Desde la década de los ochenta, el mundo ha ido caminando hacia mayores niveles de integración económica, es decir, hacia mayores grados de apertura de los mercados de bienes y de capitales. Para tener una idea mejor del grado de integración, así como de la modificación de la estructura de exportaciones, en el grupo de países seleccionados, se ha elegido una batería de indicadores extraídos de Gwartney et al., (2010) que se presenta en la Tabla 1. 
Tabla 1

Indicadores de integración económica internacional y evolución de la estructura de exportaciones

\begin{tabular}{|c|c|c|c|c|c|c|c|c|c|c|c|c|c|c|}
\hline & \multirow[b]{2}{*}{$\begin{array}{l}n \\
0 \\
2 \\
\&\end{array}$} & \multirow[b]{2}{*}{$\begin{array}{c}\% \text { ingresos } \\
\text { arancelarios } \\
\text { sobre sector } \\
\text { comercial }\end{array}$} & \multirow[b]{2}{*}{$\begin{array}{c}\text { Tarifas } \\
\text { medias } \\
\text { ponderadas } \\
(\%)\end{array}$} & \multirow{2}{*}{$\begin{array}{c}\text { Grado de } \\
\text { libertad al } \\
\text { mercado } \\
\text { internacional de } \\
\text { capitales (Nota } \\
0-10)\end{array}$} & \multirow[b]{2}{*}{$\begin{array}{c}\text { Libertad de } \\
\text { comercio } \\
\text { internacional } \\
\text { (Nota 0-10) }\end{array}$} & \multirow[b]{2}{*}{$\begin{array}{l}\text { Libertad de } \\
\text { comercio } \\
\text { internacional } \\
\text { ajustada } \\
\text { (Nota } 0-10)\end{array}$} & \multirow[b]{2}{*}{ 告 } & \multirow[b]{2}{*}{$\begin{array}{c}\text { Índice de } \\
\text { apertura } \\
(\mathrm{X}+\mathrm{M}) / \mathrm{PIB} \\
(\%)\end{array}$} & \multicolumn{6}{|c|}{ Estructura de exportaciones sobre total manufacturas } \\
\hline & & & & & & & & & $\begin{array}{l}\text { Agricultura } \\
\text { y materias } \\
\text { primas }(\%)\end{array}$ & $\begin{array}{c}\text { Alimentos } \\
(\%)\end{array}$ & $\begin{array}{c}\text { Combustibles } \\
(\%)\end{array}$ & $\begin{array}{c}\text { Alta } \\
\text { tecnología } \\
(\%)\end{array}$ & $\begin{array}{c}\text { Minerales } \\
\text { y metales } \\
(\%)\end{array}$ & $\begin{array}{c}\text { Otras } \\
\text { manufacturas } \\
(\%)\end{array}$ \\
\hline \multirow{5}{*}{ 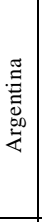 } & 1985 & 12,7 & 27,0 & 0,0 & 1,5 & 2,9 & 1985 & 13,8 & 3,5 & 66,1 & 7,3 & - & 1,7 & - \\
\hline & 1990 & 9,9 & 20,5 & 0,0 & 3,3 & 4,3 & 1990 & 11,6 & 4,3 & 56,3 & 7,8 & - & 2,4 & - \\
\hline & 1995 & 3,7 & 10,5 & 9,5 & 6,9 & 6,9 & 1995 & 15,9 & 4,3 & 49,8 & 10,3 & 3,5 & 1,6 & 30,5 \\
\hline & 2000 & 3,1 & 12,6 & 6,6 & 6,3 & 6,3 & 2000 & 18,1 & 1,8 & 43,6 & 17,6 & 9,1 & 3,0 & 24,9 \\
\hline & 2008 & 6,6 & 11,6 & 4,5 & 6,4 & 6,4 & 2007 & 38,3 & 1,2 & 50,4 & 10,9 & 6,6 & 3,8 & 27,1 \\
\hline \multirow{5}{*}{ 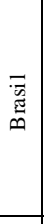 } & 1985 & 3,2 & 55,6 & 0,0 & 1,6 & 3,0 & 1985 & 17,9 & 2,6 & 36,8 & 6,4 & - & 9,4 & - \\
\hline & 1990 & 3,7 & 30,0 & 0,0 & 3,6 & 4,5 & 1990 & 11,7 & 3,4 & 27,7 & 2,2 & 7,1 & 13,6 & 46,0 \\
\hline & 1995 & 2,8 & 12,0 & 3,6 & 5,6 & 5,6 & 1995 & 13,1 & 5,2 & 28,5 & 0,9 & 4,9 & 10,3 & 50,2 \\
\hline & 2000 & 4,2 & 14,4 & 4,2 & 6,1 & 6,1 & 2000 & 17,7 & 4,8 & 23,2 & 1,6 & 18,7 & 10,1 & 41,6 \\
\hline & 2008 & 2,1 & 13,6 & 4,8 & 6,4 & 6,4 & 2007 & 21,5 & 3,8 & 26,2 & 8,3 & 12,4 & 11,8 & 37,5 \\
\hline \multirow{5}{*}{ 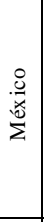 } & 1985 & 2,6 & 30,0 & 2,0 & 4,1 & 5,0 & 1985 & 24,9 & 0,9 & 8,1 & 60,1 & - & 3,8 & - \\
\hline & 1990 & 2,0 & 11,1 & 5,0 & 7,1 & 7,3 & 1990 & 32,1 & 1,6 & 11,6 & 37,6 & 8,3 & 5,7 & 35,2 \\
\hline & 1995 & 1,1 & 12,6 & 6,4 & 7,7 & 7,7 & 1995 & 54,2 & 1,3 & 7,7 & 10,3 & 15,1 & 2,9 & 62,7 \\
\hline & 2000 & 0,9 & 16,2 & 5,1 & 7,5 & 7,5 & 2000 & 60,0 & 0,6 & 4,9 & 9,7 & 22,4 & 1,3 & 61,1 \\
\hline & 2008 & 0,9 & 12,6 & 4,3 & 6,9 & 6,9 & 2007 & 55,4 & 0,4 & 5,3 & 15,7 & 17,1 & 2,7 & 58,8 \\
\hline \multirow{5}{*}{ שี } & 1985 & 10,0 & 39,5 & 2,0 & 4,7 & 5,4 & 1985 & 22,7 & 5,1 & 12,6 & 25,8 & - & 1,5 & - \\
\hline & 1990 & 3,7 & 40,3 & 5,0 & 4,0 & 4,8 & 1990 & 32,3 & 3,5 & 12,7 & 8,3 & - & 2,1 & - \\
\hline & 1995 & 1,2 & 37,5 & 4,9 & 6,4 & 6,4 & 1995 & 38,6 & 1,8 & 8,3 & 3,6 & 10,0 & 2,1 & 74,2 \\
\hline & 2000 & 1,7 & 16,3 & 2,7 & 7,2 & 7,2 & 2000 & 39,6 & 1,1 & 5,4 & 3,2 & 18,6 & 1,8 & 69,9 \\
\hline & 2008 & 0,8 & 9,6 & 2,3 & 7,4 & 7,4 & 2007 & 64,3 & 0,5 & 2,7 & 1,6 & 29,7 & 1,8 & 63,7 \\
\hline
\end{tabular}




\begin{tabular}{|c|c|c|c|c|c|c|c|c|c|c|c|c|c|c|}
\hline & \multirow[b]{2}{*}{ 辇 } & \multirow[b]{2}{*}{$\begin{array}{c}\% \text { ingresos } \\
\text { arancelarios } \\
\text { sobre sector } \\
\text { comercial }\end{array}$} & \multirow[b]{2}{*}{$\begin{array}{c}\text { Tarifas } \\
\text { medias } \\
\text { ponderadas } \\
(\%)\end{array}$} & \multirow{2}{*}{$\begin{array}{l}\text { Grado de } \\
\text { libertad al } \\
\text { mercado } \\
\text { internacional } \\
\text { de capitales } \\
\text { (Nota 0-10) }\end{array}$} & \multirow[b]{2}{*}{$\begin{array}{l}\text { Libertad de } \\
\text { comercio } \\
\text { internacional } \\
\text { (Nota } 0-10)\end{array}$} & \multirow[b]{2}{*}{$\begin{array}{l}\text { Libertad de } \\
\text { comercio } \\
\text { internacional } \\
\text { ajustada } \\
\text { (Nota 0-10) }\end{array}$} & \multirow[b]{2}{*}{ 妾 } & \multirow[b]{2}{*}{$\begin{array}{c}\text { Índice de } \\
\text { apertura } \\
(\mathrm{X}+\mathrm{M} / \mathrm{PIB}) \\
(\%)\end{array}$} & \multicolumn{6}{|c|}{ Estructura de exportaciones sobre total manufacturas } \\
\hline & & & & & & & & & $\begin{array}{l}\text { Agricultura } \\
\text { y materias } \\
\text { primas }(\%)\end{array}$ & $\begin{array}{l}\text { Alimentos } \\
(\%)\end{array}$ & $\begin{array}{c}\text { Combustibles } \\
(\%)\end{array}$ & $\begin{array}{c}\text { Alta } \\
\text { tecnología } \\
(\%)\end{array}$ & $\begin{array}{l}\text { Minerales } \\
\text { y metales } \\
(\%)\end{array}$ & $\begin{array}{c}\text { Otras } \\
\text { manufacturas } \\
(\%)\end{array}$ \\
\hline \multirow{5}{*}{$\begin{array}{l}00 \\
\overline{0} \\
0 \\
0 \\
0 \\
0 \\
0 \\
1\end{array}$} & 1985 & 0,6 & 0,0 & 10,0 & 10,0 & 9,6 & 1985 & 172,4 & 0,3 & 1,9 & 0,3 & - & 1,0 & - \\
\hline & 1990 & 0,4 & 0,0 & 10,0 & 10,0 & 9,6 & 1990 & 217,4 & 0,3 & 2,6 & 0,3 & - & 1,0 & - \\
\hline & 1995 & 0,3 & 0,0 & 9,7 & 9,7 & 9,7 & 1995 & 256,5 & 0,3 & 2,6 & 0,3 & 20,4 & 1,4 & 75,0 \\
\hline & 2000 & 0,3 & 0,0 & 9,6 & 9,8 & 9,8 & 2000 & 246,4 & 0,4 & 1,5 & 0,5 & 23,3 & 1,9 & 72,4 \\
\hline & 2008 & 0,0 & 0,0 & 8,5 & 9,5 & 9,5 & 2007 & 347,5 & 1,0 & 2,7 & 1,5 & 19,3 & 4,1 & 71,4 \\
\hline \multirow{5}{*}{ 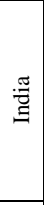 } & 1985 & 24,2 & 98,8 & 0,0 & 2,4 & 3,6 & 1985 & 10,9 & 25,3 & 25,3 & 6,0 & - & 7,6 & - \\
\hline & 1990 & 21,1 & 79,2 & 0,0 & 2,8 & 3,9 & 1990 & 13,1 & 15,6 & 15,6 & 2,9 & 2,4 & 5,2 & 58,3 \\
\hline & 1995 & 11,8 & 55,2 & 2,3 & 4,6 & 4,6 & 1995 & 18,3 & 18,7 & 18,7 & 1,7 & 4,3 & 3,6 & 53,0 \\
\hline & 2000 & 8,3 & 32,5 & 2,0 & 5,5 & 5,5 & 2000 & 20,4 & 12,9 & 12,9 & 4,3 & 5,0 & 2,9 & 62,0 \\
\hline & 2008 & 4,4 & 13,0 & 3,1 & 6,8 & 6,8 & 2007 & 30,9 & 9,2 & 9,2 & 16,2 & 5,3 & 7,8 & 52,3 \\
\hline \multirow{5}{*}{ 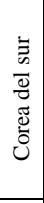 } & 1985 & 3,6 & 23,0 & 2,0 & 5,8 & 6,3 & 1985 & 63,6 & 0,8 & 4,1 & 3,1 & - & 0,7 & - \\
\hline & 1990 & 3,4 & 13,3 & 5,0 & 7,0 & 7,2 & 1990 & 51,1 & 1,3 & 3,3 & 1,0 & 17,8 & 0,8 & 75,8 \\
\hline & 1995 & 2,0 & 11,5 & 4,8 & 6,8 & 6,8 & 1995 & 50,3 & 1,3 & 2,3 & 2,0 & 25,9 & 1,0 & 67,5 \\
\hline & 2000 & 1,3 & 8,7 & 3,6 & 7,1 & 7,1 & 2000 & 62,4 & 0,9 & 1,5 & 5,4 & 34,8 & 1,2 & 56,2 \\
\hline & 2008 & 0,9 & 12,2 & 5,4 & 7,1 & 7,1 & 2007 & 69,4 & 0,8 & 0,9 & 6,6 & 33,5 & 2,5 & 55,7 \\
\hline \multirow{5}{*}{ 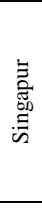 } & 1985 & 0,3 & 0,3 & 10,0 & 10,0 & 9,6 & 1985 & 276,7 & 4,4 & 8,1 & 27,0 & - & 2,2 & - \\
\hline & 1990 & 0,1 & 0,4 & 10,0 & 9,9 & 9,6 & 1990 & 308,1 & 2,6 & 5,2 & 17,9 & 39,7 & 1,6 & 33,0 \\
\hline & 1995 & 0,1 & 0,4 & 9,3 & 9,6 & 9,6 & 1995 & 288,0 & 1,1 & 3,9 & 6,8 & 53,9 & 2,0 & 32,3 \\
\hline & 2000 & 0,1 & 0,4 & 7,2 & 9,3 & 9,3 & 2000 & 293,7 & 0,5 & 2,2 & 7,4 & 62,6 & 1,1 & 26,2 \\
\hline & 2008 & 0,0 & 0,0 & 7,5 & 9,3 & 9,3 & 2007 & 336,9 & 0,3 & 1,7 & 13,8 & 46,5 & 1,7 & 36,0 \\
\hline \multirow{5}{*}{ 䨠 } & 1985 & 2,8 & - & 2,0 & 6,9 & 7,2 & 1985 & - & - & - & - & - & - & - \\
\hline & 1990 & 2,1 & 9,7 & 5,0 & 7,5 & 7,7 & 1990 & - & - & - & - & - & - & - \\
\hline & 1995 & 1,7 & 8,0 & 5,7 & 7,6 & 7,6 & 1995 & - & - & - & - & - & - & - \\
\hline & 2000 & 1,5 & 8,8 & 7,6 & 8,1 & 8,1 & 2000 & - & - & - & - & - & - & - \\
\hline & 2008 & 0,5 & 6,1 & 6,1 & 7,6 & 7,6 & 2007 & - & - & - & - & - & - & - \\
\hline
\end{tabular}


Los tres países latinoamericanos estudiados, Argentina, Brasil y México, experimentaron un progresivo proceso de liberalización comercial que puede ser observado por la evolución de sus tres principales indicadores. La evolución de la tasa de ingresos arancelarios sobre los ingresos por exportaciones muestra una fuerte reducción en Argentina aunque cae de forma moderada en Brasil y México. Las tarifas medias ponderadas se redujeron drásticamente en Brasil, pasando de un tarifa media de $55 \%$ a mediados de los ochenta a una tarifa de algo menos del $14 \%$ a mediados de los 2000. Por su parte, el aumento del grado de apertura resulta especialmente significativo en México, que pasó de un $25 \%$ en 1985 a un $55 \%$ en 2007. Las notas obtenidas respecto al grado de libertad del comercio son siempre progresivas para los tres países, pasando de niveles iniciales muy bajos (notas entre 1 y 2 en los casos de Argentina y Brasil) a niveles sensiblemente elevados (notas entre 6 y 7). Tendencias similares fueron seguidas en los mercados de capitales. Las notas registradas son visiblemente más elevadas a lo largo de los periodos, aunque el momento de mayor liberalización se registra a mediados de los noventa en Argentina y México.

El proceso de liberalización comercial afectó a la estructura de exportaciones de estos países (Tabla 1). Argentina, a pesar de mantener la fuerte concentración en Alimentos, fue reduciendo su participación en este tipo de productos y diversificando su cartera de exportaciones hacia combustibles y otras manufacturas, mejorando también la parcela relativa a la exportación de productos de Alta Tecnología. Brasil siguió un proceso de diversificación moderado. Hubo un cierto mantenimiento de la cuota de exportaciones relativa a Agricultura y Materias primas, pero también una reducción relativa a Alimentos y un aumento en Minerales y Metales, productos de Alta Tecnología y Combustibles, donde la tendencia se presume que será fuertemente creciente tras la explotación de los yacimientos del pre-sal. México mantuvo sus cuotas en Agricultura, Alimentos, Minerales y Metales. El principal cambio observado es la fuerte reducción de la concentración de la cuota de exportaciones de Combustibles y un mayor aumento de las exportaciones de productos de alta Tecnología y otras manufacturas, lo que sin duda responde a las nuevas 'maquilas' de los sectores de Transporte y de Electrónica de Consumo de multinacionales estadounidenses.

En el conjunto de países asiáticos se perciben tendencias de liberalización comercial semejantes. Todos los países redujeron sus ingresos arancelarios por volumen de comercio. Las tarifas medias registraron reducciones importantes y de carácter drástico en el caso de la India, que pasó de casi un 99\% a un 13\% o China, que pasó de casi un $40 \%$ a unas tarifas medias cercanas al 10\%. Hong-Kong y Singapur ya eran países fuertemente liberalizados (con tarifas medias e ingresos arancelarios sobre exportación próximos a cero) desde mediados de los ochenta y mantuvieron este estatus hasta la actualidad. Los índices de apertura aumentaron extraordinariamente en los casos de China e India que partían de niveles 
relativamente reducidos. En estos países, este índice llega a multiplicarse casi por 3. Hong-Kong y Singapur, con elevados índices de apertura ya desde los ochenta, experimentaron fuertes aumentos. Corea del Sur también aumentó su apertura, aunque de forma mucho más moderada.

Las mismas tendencias son observadas en las notas obtenidas sobre libertad de comercio. India comenzó el periodo con notas muy bajas (alrededor de 2) y fue aumentándolas hasta casi 7 en el 2007. China y Corea del Sur partieron de notas no tan bajas (entre 4 y 6) y mostraron tendencia creciente hasta notas que alcanzaron valores en la actualidad también en torno a 7. Taiwán comenzó con un nivel de liberalización mayor (en torno a 7) y finalizó con una mejora moderada. Singapur y Hong-Kong ya comenzaron el periodo con nota 10 o próximas a 10 y mantuvieron este estatus a lo largo del periodo.

La evolución de la internacionalización en los mercados de capitales sigue tendencias muy similares a las del comercio. Corea del Sur y Taiwán, que partieron de notas próximas a 2, lograron las notas más elevadas en 2007. India también registró cambios substantivos $\mathrm{y}$, aunque su nota actual no es muy elevada (en torno a 3), el país partió de una situación inicial muy cerrada al exterior con nota igual a 0. Singapur y Hong-Kong partieron de una situación de prácticamente completa liberalización de capitales en los ochenta y a lo largo del periodo registraron tendencias contrarias, esto es, un leve aumento de sus controles de capitales hasta notas en torno a 9,5 .

Las estructuras de comercio en los países asiáticos también registraron modificaciones. China redujo fuertemente su cuota de exportaciones en Combustibles y Alimentos y aumentó drásticamente su cuota de productos de alta tecnología. Hong-Kong mantuvo su estructura de comercio, fuertemente concentrada en Otras manufacturas y mostró un leve aumento de la importancia relativa de productos de alta tecnología. India redujo drásticamente las cuotas de exportaciones ocupadas por Agricultura, Materias primas y Alimentos, al tiempo que aumentó la participación de Combustibles y, moderadamente, la de productos de alta tecnología. Corea del Sur redujo la participación en Otras manufacturas y aumentó fuertemente su concentración en productos de alta tecnología. Finalmente, Singapur redujo sus cuotas de exportación en 4 de las 6 categorías de productos observadas para concentrarse fuertemente en productos de alta tecnología, los cuales llegaron a ocupar a principios de los 2000 el $60 \%$ de sus exportaciones totales de manufacturas.

En conjunto puede decirse que los países asiáticos han alcanzado mayores niveles de liberalización comercial que los latinoamericanos, aunque las diferencias no son especialmente relevantes. Los latinoamericanos, por su parte, han registrado relativamente mayores niveles de liberalización de capitales que China e India, aunque no cuando se compara con Corea del Sur, Taiwán, Hong-Kong y Singapur, 
dado que estos dos últimos ya se encontraban altamente integrados con el mundo desde el periodo considerado como de pre-apertura.

Con respecto a la estructura de comercio, se confirma que los países asiáticos tendieron a una mayor concentración de su patrón de exportación en otras manufacturas y en productos de alta tecnología, mientras que los países latinoamericanos (Argentina y Brasil) tendieron a concentrarse en productos vinculados a la explotación de recursos naturales. México se reveló como una excepción a esta tendencia como consecuencia de las actividades de ensamblaje realizadas por las empresas estadounidenses vinculadas a estas actividades, lo que no significa necesariamente una correspondencia con especialización productiva dado el escaso valor añadido que este tipo de actuación crea en el país receptor.

\section{Especialización tecnológica, captura e integración económica}

\subsection{Fuentes de información}

Este trabajo utiliza datos de patentes depositadas en la Oficina Europea de Patentes (EPO) entre 1985 y 2008 disponibles en las bases de datos de Space Bulletin de la propia EPO. La nacionalidad de la patente se corresponde con la residencia del inventor (Zeebroeck et al., 2006). Se trabajó con un nivel de desagregación de 29 clases tecnológicas agregadas a partir de 4 dígitos siguiendo la metodología del Fraunhofer Institute for Systems and Innovation Research y utilizado por Mancusi (2003). A pesar de que otros trabajos sobre ETN seleccionan apenas patentes depositadas por empresas, este trabajo considera todas las patentes independientemente del agente que realiza el depósito, asumiendo implícitamente la idea de que la ETN debe reflejar la base técnica de un país, la cual no incluye sólo empresas, sino también universidades, centros públicos de investigación, agencias de gobierno e investigadores independientes (Brusoni; Geuna, 2003).

Las patentes son ampliamente utilizadas en la literatura para el análisis de competencias porque proveen de datos detallados de forma regular y para largos períodos de tempo, porque pueden ser agrupadas por empresa, localización geográfica y campos técnicos y porque representan resultados de innovación de esfuerzos formales e informales (Patel; Pavitt, 1991). Sin embargo, hay también algunos límites en el uso de patentes como fuente de información para construir indicadores sobre especialización tecnológica. En primer lugar, porque las patentes revelan distribuciones de competencias entre campos técnicos, pero no distribuciones de capacitaciones en el sentido de Archibugi y Coco (2005). Las medidas de capacitaciones deberían incluir indicadores de conocimiento incorporado y desincorporado, codificado y tácito, así como de difusión y generación de conocimiento. Las patentes sólo dan información sobre tecnologías desincorporadas y sobre conocimiento codificado. Por tanto, incluso asumiendo que existen complementariedades entre las tres categorías, el uso exclusivo de 
patentes subestima el conjunto de aspectos que transforma una competencia en una capacitación. En segundo lugar, las patentes subestiman la contribución de las bases científicas a la creación de bases técnicas debido a la distancia natural que existe entre la ciencia básica y las capacitaciones en ingeniería que permiten transformar los resultados científicos en productos rentables (Brusoni; Geuna, 2003). Por tanto, es posible que un país tenga fuertes competencias y capacitaciones en 'desarrollo' que se encuentran débilmente apoyadas en conocimiento básico propio. En tercer lugar, medir la especialización tecnológica relativa a productos específicos a industrias puede implicar el uso de clasificaciones tecnológicas que no respondan a las usuales clasificaciones de patentes. Y, en cuarto lugar, algunas competencias tecnológicas nacionales pueden ser subestimadas cuando éstas son construidas sobre tecnologías (o bases de conocimiento) no patentables o sobre tecnologías que no pueden ser protegidas mediante patentes.

La EPO presenta algunas ventajas en comparación con la USPTO (oficina americana de patentes) (Le Bas; Sierra, 2002; Grupp; Schmoch, 1999; Zeebroeck et al., 2006). En primer lugar, la EPO es la oficina de patentes más internacionalizada del mundo, porque una patente es potencialmente extensible a todos los países firmantes de la Convención de Münich. Esto significa que no existe un sesgo hacia un determinado país, o sea, no hay "efecto doméstico", mientras que la UPSTO, al ser válida apenas dentro del territorio de los Estados Unidos, contiene el sesgo de las estrategias de cómo patentar dirigidas a los mercados estadounidenses. En segundo lugar, las tasas pagadas por la solicitud de una patente en la EPO son muy elevadas, actuando como un filtro económico porque tiende a eliminar patentes de bajo valor industrial. En tercer lugar, la EPO publica patentes garantizadas y depositadas 18 meses después de la solicitud, mientras que la UPSTO sólo lo hace después de dos años (en media).

Los países latinoamericanos contemplados para el estudio fueron Argentina, Brasil y México. Otros países no fueron incluidos por su escaso número de depósitos en la EPO. Los periodos considerados como de pre y post apertura fueron 1985-1995 y 1999-2008. Con el objetivo de mantener homogéneos los periodos pre y post apertura para todos los países y dada la dificultad de establecer un año común a todos los países que marcase el punto de inflexión en su proceso de internacionalización, se consideró como año de referencia 1995, es decir, la mitad de la década de los noventa. Hasta finales de los noventa los procesos de internacionalización todavía estaban en fase de maduración en varios de los países contemplados, lo que justifica que para el inicio del periodo post-integración se haya escogido el año de 1999. El periodo de 10 años (hacia adelante y hacia atrás) fue el que permitió la base de datos del Space Bulletin de 2009.

Los países asiáticos considerados fueron China, India, Corea del Sur, Singapur, Taiwán y Hong-Kong, para los mismos periodos de pre y post abertura. 
El área de integración considerada en el mundo. A pesar de la participación de México en la Nafta y de Argentina y Brasil en el Mercosur, estas áreas de integración no fueron consideradas por la fuerte asimetría entre países dentro de cada área, lo que se reduciría el análisis de la especialización y convergencia a relaciones bilaterales entre México y Estados Unidos en el caso de la Nafta o entre Brasil y Argentina con respecto a ellos mismos en el caso de Mercosur.

\subsection{Desarrollo tecnológico, convergencia y especialización tecnológica}

Dos ejercicios revelarán la evidencia acerca de los ritmos de captura y sobre los cambios registrados en las ETN. El primero es la estimación del dinamismo tecnológico a través del cálculo del crecimiento de la cuota de patentes que cada país registró entre periodos y su descomposición a través de un análisis shift-share (Laursen, 1999). El segundo es la observación de la evolución de los patrones de especialización tecnológica a través del indicador de convergencia propuesto por Archibugi y Pianta (1992, cap. 8) y Pianta y Meliciani (1996) y los de especialización usualmente utilizados para el comercio por la Unctad.

Denotando $p_{j}$ como la cuota de patentes del país $j$-ésimo en el mundo; $p_{i j}$ la cuota de patentes del país $j$-ésimo en el campo técnico $i$-ésimo; $o_{i}$, la cuota de patentes del campo técnico i-ésimo en el mundo; y (t-1) el periodo inicial de análisis, el crecimiento de la cuota de patentes de un país $\left(p_{j}\right)$ puede ser descompuesto en cuatro componentes (Laursen, 1999);

$$
\Delta p_{j}=\sum_{i}\left(\Delta p_{i j} o_{i}^{t-1}\right)+\sum_{i}\left(p_{i j}^{t-1} \Delta o_{i}\right)+\sum_{i}\left(\Delta p_{i j} \frac{\left(\Delta o_{i}+\left|\Delta o_{i}\right|\right)}{2}\right)+\sum_{i}\left(\Delta p_{i j} \frac{\left(\Delta o_{i}-\left|\Delta o_{i}\right|\right)}{2}\right)
$$

El primer sumando representa el efecto cuota tecnológica o la parte del crecimiento que se debe al dinamismo de su actividad patentadora en sentido estricto (tecnológica en sentido amplio), manteniendo constante el peso del campo técnico del período inicial. El segundo sumando recoge el efecto tecnológico estructural o la parte del crecimiento que se debe a un "correcto" (o "incorrecto") patrón de especialización tecnológica inicial, es decir, si en el periodo inicial el país se encontraba especializado (o des-especializado) en campos técnicos que se mostraron dinámicos (o estancados) entre los períodos observados. Este segundo sumando se interpreta como una medida del efecto de la OT sobre el crecimiento de la cuota de patentes de un país, dado que el crecimiento sectorial de patentes representa una medida del dinamismo de la oportunidad tecnológica $(\mathrm{OT})$ entre periodos ${ }^{2}$. El tercer y cuarto sumando conforman el efecto adaptación. Cuando toma valor negativo, significa que el país salió de sectores de OT dinámica o entró en sectores que se mostraron de OT estancada; y cuando toma valor positivo

(2) A partir de esta definición se considera que un campo técnico es de OT dinámica cuando el crecimiento de su cuota de patentes entre periodos es positiva y estancada si es negativa. 
significa que el país pasó a ser activo en tecnologías con OT dinámica o abandonó la actividad patentadora sobre tecnologías cuya OT se mostró estancada entre los periodos observados.

El indicador más habitualmente utilizado para medir la convergencia tecnológica es la chi-cuadrado $\left(\chi^{2}\right)$. La $\chi^{2}$ es una medida de la distancia (en términos porcentuales) entre la estructura tecnológica de cada país y su área de referencia y se calcula como $\chi^{2}=\sum_{i=1}^{n} \frac{(p i j-p i w)^{2}}{p i w}$, donde $p_{i j}$ sigue la anterior notación y $p_{i w}$ es la cuota mundial de patentes del área técnica $i$ (Archibugi; Pianta, 1992, cap. 8; Pianta; Meliciani, 1996). Este indicador toma valor 0 cuando la similitud es total y aumenta a medida que son mayores las distancias entre cada país y su área de referencia.

Los indicadores de especialización utilizados por la Unctad son dos; uno absoluto y otro relativo. El absoluto es el índice de concentración normalizado de Hirschmann para valores entre 0 and 1 , es decir, $H_{j}=\frac{\sqrt{\sum_{i=1}^{n} p_{i j}-\sqrt{1 / n}}}{1-\sqrt{1 / n}}$. El relativo es el llamado 'índice de diversificación' $\left(S_{j}\right)$ definido como $S_{j}=\frac{\sum_{i=1}^{n}\left|p_{i j}-p_{i W}\right|}{2}$, donde $p_{i j} \mathrm{y} p_{i w}$ siguen las notaciones anteriores.

Los resultados de este conjunto de indicadores se presentan en la Tabla 2. Los países latinoamericanos registraron crecimientos elevados de su cuota de patentes en el mundo siendo las mayores las registradas por Brasil $(157,2 \%)$. Este crecimiento se debió a un elevado dinamismo tecnológico que compensó una especialización inicial desfavorable en los casos de Brasil y México. Sin embargo, y en consonancia con los trabajos de Montobbio y Rampa (2005), estos países se dirigieron hacia campos técnicos de bajo dinamismo entre periodos, lo cual se revela en el signo negativo del efecto adaptación. Los países asiáticos mostraron también elevados crecimientos de su cuota de patentes en el mundo, salvo en el caso de Hong-Kong que registró estancamiento. Los mayores crecimientos fueron registrados por China $(1193,2 \%)$, Corea del Sur $(1099,1 \%)$ e India $(718,8 \%)$. Esta tendencia es también el resultado de un elevado dinamismo tecnológico, más que de una "correcta" especialización inicial que en los casos de China e India no era favorable. A diferencia del grupo de los países latinoamericanos, algunos países asiáticos presentan una movilidad hacia campos técnicos con OT dinámica entre periodos, o sea, un aprovechamiento de oportunidades tecnológicas que es especialmente relevante en China y Corea del Sur. India y Hong-Kong no presentaron esta capacidad de aprovechamiento de OT dirigiéndose hacia sectores estancados o saliendo de los dinámicos, lo cual se revela en el signo negativo del efecto adaptación (tercera columna de la Tabla 2). 
Tabla 2

Crecimiento, convergencia y especialización tecnológica entre periodos pre y post apertura

\begin{tabular}{|c|c|c|c|c|c|c|c|}
\hline \multirow{2}{*}{ (*); coeficientes de correlación } & \multirow{2}{*}{$\begin{array}{c}\text { Crecimiento } \\
\text { dentreareriofos } \\
(\%)\end{array}$} & \multicolumn{3}{|c|}{ Descomposición del crecimiento (\%) } & \multirow{2}{*}{$\begin{array}{l}\text { Convergencia }\left(\chi^{2}\right) \\
\text { Tasa de crecimiento }\end{array}$} & \multirow{2}{*}{$\begin{array}{l}\text { Especialización (\%) } \\
\text { Tasa de crecimiento }\end{array}$} & \multirow{2}{*}{$\begin{array}{c}\text { Concentración } \\
\text { Tasa de crecimiento } \\
\end{array}$} \\
\hline & & E. Tecnológico & E. Estructural & E. Adaptación & & & \\
\hline Argentina & 144.1 & 152.09 & 0.57 & -8.55 & 34.5 & 38.0 & 11.3 \\
\hline Brasil & 157.2 & 184.27 & -2.73 & -24.39 & 20.5 & 9.0 & -0.5 \\
\hline México & 146.2 & 169.97 & -1.01 & -22.79 & -27.3 & -17.8 & -8.6 \\
\hline China & 1193.2 & 1068.91 & -2.36 & 126.66 & 88.6 & 19.7 & 62.5 \\
\hline Corea del Sur & 1099.1 & 934.56 & 15.88 & 148.66 & 21.7 & 22.6 & 31.0 \\
\hline Hong-Kong & 0.3 & 5.40 & 4.49 & -9.55 & 6.2 & -15.6 & -6.2 \\
\hline India & 718.8 & 745.12 & -7.22 & -19.10 & -21.8 & -13.6 & -10.9 \\
\hline Singapur & 407.3 & 355.28 & 10.81 & 41.25 & -32.4 & -15.8 & 4.1 \\
\hline Taiwán & 211.7 & 193.67 & 3.92 & 14.16 & -53.7 & -26.3 & -1.3 \\
\hline Correlación* con convergencia & 0.501640 & 0.489899 & 0.062924 & 0.862616 & & - & \\
\hline Correlación* con crecimiento & & & & & 0.501640 & 0.382261 & 0.778032 \\
\hline
\end{tabular}

Fuente: Elaboración propia a partir de informaciones contenidas en el Space Bulletin, 2008. European Patent Office. 
El dinamismo tecnológico confirma que hubo captura. La cuestión es si la captura llevó a estos países a una mayor aproximación o a un mayor distanciamiento de sus perfiles tecnológicos con el mundo, lo que significaría diferentes patrones de competencia tecnológica en la arena internacional. Si los países convergen hacia las mismas áreas tecnológicas (similitud de las distribuciones), el escenario será de competencia por tecnologías. Si los países se especializan en campos técnicos diferentes (mayor distancia de las distribuciones), el escenario será de complementariedad y cooperación tecnológica (Archibugi; Pianta, 1994).

Los resultados a este respecto pueden ser observados en las tres últimas columnas de la Tabla 2. Entre los países latinoamericanos pueden observarse diferentes tendencias. Argentina y Brasil registraron un aumento de su distancia con el mundo (divergencia), mientras que México registró convergencia. El distanciamiento tecnológico de Brasil se debe básicamente al elevado aumento de las distancias en tecnologías de bienes de consumo, altamente concentradas en empresas multinacionales de electrónica de consumo como Whirlpool, Electrolux, Unilever PCL o Brastemp, y en ingeniería química, concentrando el $25 \%$ de las distancias mantenidas con el mundo. Otros campos técnicos responsables de este mayor distanciamiento, pero de menor importancia, son transporte, alimentos y agricultura. En el caso argentino, los campos técnicos que empujaron al mayor distanciamiento fueron aparatos electrónicos e ingeniería eléctrica, biotecnología y bienes de consumo (conducido, en su mayor parte, por empresas multinacionales no residentes), así como por la fuerte concentración de esfuerzos tecnológicos en el periodo post-apertura (un 37\%) en tecnologías relacionadas con farmacéutica y cosmética, cuya actividad se encuentra muy distribuida entre empresas nacionales e internacionales. México, por su parte, aumentó fuertemente sus distancias con el mundo en tecnologías de alimentos y agricultura, cuya actividad está conducida por algunas empresas internacionales, pero también por centros públicos de investigación. Sin embargo, este distanciamiento se vio compensado por importantes reducciones de las distancias en tecnologías de procesamiento de materiales, química orgánica, procesamiento de alimentos, tecnologías ambientales, química macromolecular y polímeros, aparatos electrónicos e ingeniería eléctrica o en tecnologías de control y medida.

En el grupo de países asiáticos, se observan también tendencias contrapuestas. China y Corea del Sur aumentan fuertemente sus distancias tecnológicas con el mundo. Hong-Kong también registra distanciamiento aunque de forma más moderada. La tendencia observada en el caso de China se deriva de una elevada actividad dirigida a tecnologías audiovisuales y de telecomunicaciones, la cual concentra el 65,6\% de su desvío con el patrón mundial en el periodo post-integración. En este sector, apenas una única empresa nacional, 
Huwaei Technologies Corporation, LTD, fue responsable del 50\% de las patentes depositadas. Corea del Sur también aumentó la concentración de sus esfuerzos tecnológicos en tecnologías audiovisuales y de telecomunicaciones, siendo ésta un área donde ya estaba fuertemente especializada antes de la integración. El desvío que registra el país respecto al mundo en esta tecnología representa el $61 \%$ de su distanciamiento total en el periodo post-integración. Dos empresas nacionales son responsables por esta tendencia: Samsung, con el $62 \%$ de las patentes y LG, con el $24 \%$, es decir, ambas absorbieron el $86 \%$ de las patentes de un único campo técnico en el periodo post-integración.

Para examinar el grado de asociación entre dinamismo tecnológico (creación y captura) con convergencia, fueron calculados coeficientes de correlación de Pearson entre cada componente del crecimiento y la tasa de variación de la $\chi^{2}$. Un signo positivo de la correlación entre el efecto cuota tecnológica y la tasa de variación de la $\chi^{2}$ significa que el dinamismo tecnológico está positivamente asociado con divergencia. Una asociación positiva entre la tasa de variación de la $\chi^{2}$ y el componente estructural significa que cuanto más favorable haya sido el posicionamiento de países en áreas que se presentaron con OT dinámicas, mayor es la divergencia tecnológica, esto es, el efecto de la OT sobre determinadas áreas dinámicas conduciría los esfuerzos tecnológicos de los países a una mayor concentración en esas áreas (y sus vinculadas). Finalmente, una asociación positiva entre el crecimiento de la $\chi^{2}$ y el efecto adaptación significa que un movimiento hacia tecnologías de OT dinámica o abandono de tecnologías estancadas se corresponde con divergencia y viceversa. En los tres casos los coeficientes son positivos, aunque sólo se muestra significativo para el efecto adaptación y, en menor medida, para el efecto tecnológico. El efecto estructural asociado a 'correctas' o 'incorrectas' especializaciones iniciales parece no jugar ningún papel, tanto en lo que se refiere al dinamismo tecnológico ulterior como en la determinación de los ritmos de convergencia. Estos resultados indican que, para este nivel de desagregación, la captura de este conjunto de países seguidores se ha caracterizado más por un aumento de las distancias mantenidas con el mundo, incluso cuando ésta ha propiciado la extensión de la base técnica hacia campos técnicos que contaban con muy poca (o ninguna) actividad inicial. Este efecto también se puede observar a través de la evolución de los indicadores de especialización y concentración. Brasil, Argentina, China y Corea del Sur aumentaron su especialización entre periodos, aunque sólo ésta va acompañada del aumento de la concentración de esfuerzos nacionales en los casos de Argentina, China y Corea del Sur. El resto de los países tendió a diversificar su base técnica. La tendencia a la diversificación tecnológica va acompañada de una también mayor distribución de los esfuerzos tecnológicos nacionales en los casos de México, Hong-Kong, India y Taiwán. Sin embargo, en términos agregados, el dinamismo 
tecnológico se asocia positiva y significativamente con concentración, dada la fuerte tendencia observada en los asiáticos China y Corea del Sur.

En resumen, parece haber evidencia de que existe una cierta tendencia por parte de los países latinoamericanos a especializarse en tecnologías relacionadas con la explotación de recursos naturales, en cuanto que en los asiáticos, el patrón tecnológico gravita hacia tecnologías de telecomunicaciones y audiovisual fuertemente marcado en los casos de China y Corea del Sur. Sin embargo, no parece haber evidencia de que algún patrón de especialización tecnológica inicial haya representado un límite al proceso de captura en términos de dinamismo tecnológico. La captura ha permitido un patrón de movilidad tecnológica que ha conducido a un escenario internacional de divergencia (busca por la complementariedad tecnológica) y a una concentración de las especializaciones en los casos de Argentina China y Corea del Sur. En el resto de países asiáticos y en México se observa un patrón de convergencia (competencia por tecnologías) acompañado de una diversificación de la base técnica.

\subsection{Captura y cambio de las estructuras tecnológicas}

Los resultados anteriores indican que los procesos de captura pueden acontecer independientemente de los posicionamientos iniciales 'correctos' o 'incorrectos'. El dinamismo tecnológico parece estar más asociado a la generación de capacidad de absorción (estimulado o no por políticas públicas) o a una reconducción de la base productiva que impulsó el desarrollo de nuevas competencias tecnológicas o extendió las pre-existentes. En este sentido, el análisis de movilidad ayuda a comprender cómo los países han seguido el proceso de construcción de sus competencias tecnológicas actuales. Si fueron creadas sobre competencias previas, las hipótesis sobre acumulación tecnológica prevalecen. Sino, el proceso de creación de competencias deberá estar más asociado a factores de carácter estructural o al estímulo de políticas públicas en el fortalecimiento de los SNI.

Las competencias tecnológicas pueden clasificarse en cuatro categorías: centrales (c), de fondo (f), nicho (h) y marginales (g) (Patel; Pavitt, 1997). Las competencias centrales representan las fortalezas tecnológicas de un país. Las competencias nicho son tecnologías donde el país tiene especialización, pero donde la intensidad tecnológica es relativamente poco significativa. Las competencias de fondo, representan tecnologías donde el país no detenta especialización, pero donde asigna relativamente una cantidad de recursos a la innovación por encima de la media de entre todas las posibilidades tecnológicas. Las competencias nicho y de fondo representan trayectorias naturales de crecimiento tecnológico y, por tanto, movilidad esperada, ya que a partir de ellas pueden construirse competencias centrales. Las nicho, porque explotan nichos tecnológicos a partir del conocimiento 
previo donde existe capacidad de nueva creación. Las de fondo, porque representan esfuerzos en adquirir capacidad de absorción (captura) en áreas técnicas donde el país no está especializado, pudiendo llevar al desarrollo y especialización de tecnologías relacionadas. Las competencias marginales son tecnologías donde no existe ni especialización ni una asignación de recursos a la innovación relevante. La caracterización de campos técnicos en cada una de las anteriores categorías fue realizada combinando los valores de la Ventaja Tecnológica Revelada -VTR(definida como $\frac{p_{i j}}{p_{i W}}$, siguiendo la anterior notación, que cuando toma valores mayores que 1 indica especialización y cuando toma valores menores que 1 indica des-especialización) con valores superiores o inferiores al valor medio equivalente a una distribución homogénea de pesos $\left(\mathrm{s}_{\mathrm{i}}\right)$ igual a $1 / \mathrm{n}$ con $\mathrm{n}=29$ clases tecnológicas como indicador del esfuerzo relativo en cada tecnología (Cuadro 1).

Cuadro 1

Condiciones de especialización y esfuerzo tecnológico para clasificar competencias

\begin{tabular}{|c|c|c|c|}
\hline \multirow{2}{*}{} & \multicolumn{2}{|c|}{ VTR $_{\mathrm{i}}$} \\
\cline { 3 - 4 } & $>1 / \mathrm{n}$ & Central (c) & De Fondo (f) \\
\hline \multirow{3}{*}{$\mathrm{s}_{\mathrm{i}}$} & $<1 / \mathrm{n}$ & Nicho (h) & Marginal (g) \\
\cline { 2 - 4 } & & & \\
\hline
\end{tabular}

Una vez caracterizadas las clases tecnológicas en términos de competencias, serán elaborados los indicadores que definen la persistencia y movilidad agregadas para cada país de acuerdo con la evolución de tal caracterización. Cuando una clase tecnológica permanece caracterizada por el mismo tipo de competencia entre periodos, se considera que hay persistencia. En caso contrario, se considera que hay movilidad (Cuadro 2).

Los indicadores de persistencia y movilidad serán calculados considerando el número de campos técnicos y el número de patentes registradas por campo técnico. Así, la persistencia agregada en términos de clases tecnológicas $\left(P_{j}^{T}\right)$ se define como el porcentaje de las $m$ clases tecnológicas que permanecen en la misma categoría entre los periodos anterior y posterior a la integración sobre el total de las $n$ clases tecnológicas contempladas. Designando $j$ al país; $i$ a la distribución de competencias antes de la integración $(i=c, h, f, g) ; k$, a la distribución de competencias después de la integración $(k=c, h, f, g)$ y $T_{i k j}$ el número de campos técnicos caracterizados como $i$ en el periodo inicial y como $k$ en el final, la persistencia agregada se define como $P_{j}^{T}=\frac{\sum_{i k k} T_{i k j}}{n}$ cuando $i=k$. En términos de patentes $\left(P_{j}^{P}\right)$, el indicador recoge el porcentaje de patentes registradas por un país en el periodo post-integración en las $m$ clases tecnológicas que 
persistieron entre periodos sobre o total de patentes registradas en el periodo postintegración $\left(P_{j}^{p}=\frac{\sum_{i k k} p_{i k j}}{p_{j}}\right)$, siendo $P_{i k j}$ el número de patentes del país $j$ en el periodo post-integración relativas a las $T_{i k j}$ clases tecnológicas cuando $i=k$.

\section{Cuadro 2}

Definición de persistencia y descomposición de la movilidad de competencias para cada campo técnico entre periodos

\begin{tabular}{|c|c|c|c|c|c|}
\hline & \multicolumn{5}{|c|}{ Distribución de competencias después de la integración $(k)$} \\
\hline & & Central & Nicho & De fondo & Marginal \\
\hline \multirow{4}{*}{$\begin{array}{l}\text { Distribución } \\
\text { competencias } \\
\text { antes de la } \\
\text { integración } \\
\text { (i) }\end{array}$} & Central & $\begin{array}{c}\text { Persistencia } \\
\text { especialización }\end{array}$ & $\begin{array}{l}\text { Movilidad no } \\
\text { Esperada } \\
\text { (destrucción) }\end{array}$ & $\begin{array}{l}\text { Movilidad no } \\
\text { Esperada } \\
\text { (destrucción) }\end{array}$ & $\begin{array}{l}\text { Movilidad no } \\
\text { Esperada } \\
\text { (destrucción) }\end{array}$ \\
\hline & Nicho & $\begin{array}{l}\text { Movilidad } \\
\text { Esperada }\end{array}$ & $\begin{array}{c}\text { Persistencia } \\
\text { especialización }\end{array}$ & $\begin{array}{l}\text { Movilidad no } \\
\text { Esperada } \\
\text { (destrucción) }\end{array}$ & $\begin{array}{l}\text { Movilidad no } \\
\text { Esperada } \\
\text { (destrucción) }\end{array}$ \\
\hline & De fondo & $\begin{array}{l}\text { Movilidad } \\
\text { Esperada }\end{array}$ & $\begin{array}{l}\text { Movilidad } \\
\text { Esperada }\end{array}$ & $\begin{array}{c}\text { Persistencia } \\
\text { des- } \\
\text { especialización }\end{array}$ & $\begin{array}{l}\text { Movilidad no } \\
\text { Esperada } \\
\text { (destrucción) }\end{array}$ \\
\hline & Marginal & $\begin{array}{l}\text { Movilidad no } \\
\text { Esperada } \\
\text { (construcción) }\end{array}$ & $\begin{array}{l}\text { Movilidad no } \\
\text { Esperada } \\
\text { (construcción) }\end{array}$ & $\begin{array}{l}\text { Movilidad no } \\
\text { Esperada } \\
\text { (construcción) }\end{array}$ & $\begin{array}{c}\text { Persistencia } \\
\text { des- } \\
\text { especialización }\end{array}$ \\
\hline
\end{tabular}

La persistencia de la especialización se mide como $P S_{j}^{T}=\frac{\sum_{i k} T_{i k j}}{n} \mathrm{y}$ $P S_{j}^{p}=\frac{\sum_{i k k} p_{i k j}}{P_{j}}$ ), siendo $P_{i k j}$ el número de patentes relativas a las $T_{i k j}$ clases tecnológicas en los casos en que $i=c, h$ y $k=c, h$ cuando $i=k$. La persistencia en la des-especialización se mide utilizando los mismos indicadores $P D_{j}^{T}=\frac{\sum_{i, k} T_{i k j}}{n} \mathrm{y}$ $\left.P D_{j}^{p}=\frac{\sum_{i \bar{k}} p_{i k j}}{P_{j}}\right)$, siendo $P_{i k j}$ el número de patentes relativas a las $T_{i k j}$ clases tecnológicas con $i=f, g$ y $k=f, g$ siempre que $i=k$ (Cuadro 2).

La movilidad agregada $\left(M_{j}^{T}\right)$ se mide como el porcentaje de las $n-m$ clases tecnológicas que cambian de categoría entre periodos, es decir, $M_{j}^{T}=\frac{\sum_{i k} T_{i k j}}{n}$ con $i \neq k$. En términos de patentes, el indicador $\left(M_{j}^{P}\right)$ recoge el porcentaje de patentes registradas por un país en las $n-m$ clases tecnológicas que pasaron a ser competencias diferentes entre periodos sobre o total de patentes registradas $\left(P_{j}\right)$ en el periodo post-integración, es decir, $M_{j}^{P}=\frac{\sum_{i k} p_{i k j}}{P_{j}}$, siendo $P_{i k j}$ el número de patentes relativas a las $T_{i k j}$ clases tecnológicas con $i \neq k$. 
La movilidad agregada puede ser descompuesta en tres componentes. El primero, llamado "movilidad esperada", es el que se refiere al efecto de trayectoria natural del cambio técnico a través de los procesos de acumulación y aprendizaje relativos a esfuerzos pasados. Manteniendo la misma notación, este efecto es medido en términos del número de clases tecnológicas $M E_{j}^{T}=\frac{\sum_{i k} T_{i k j}}{n}$ y del número de patentes $M E_{j}^{p}=\frac{\sum_{i k} p_{i k j}}{p_{j}}$, siendo en ambos casos $i=h, f$ y $k=c, h$ con $i \neq j$.

Los otros dos componentes son efectos 'no esperados', es decir, son efectos observados que no pueden ser explicados por la teoría de la acumulabilidad y que se derivan de cambios drásticos en la estructura tecnológica, probablemente más asociados a la captura inducida y al cambio estructural. El segundo componente es un efecto positivo y se refiere a la creación de competencias centrales, nicho o de fondo a partir de competencias marginales. Este efecto es medido también en términos del número de clases tecnológicas como $M N E C_{j}^{T}=\frac{\sum_{i k} T_{i k j}}{n}$ y del número de patentes $M N E C_{j}^{p}=\frac{\sum_{i k} p_{i k j}}{p_{j}}$, donde en ambos casos $i=g$ y $k=c, h, f$ (cuadro 2). El tercer componente es un efecto perverso que puede acompañar a los procesos de integración y que consiste en la destrucción de competencias creadas en el pasado, lo que significa un verdadero cambio estructural. Este efecto es medido en términos del número de clases tecnológicas como $\quad M N E D_{j}^{T}=\frac{\sum_{i k} T_{i k j}}{n}$ y en términos del número de patentes como $M N E D_{j}^{p}=\frac{\sum_{i k} p_{i k j}}{p_{j}}$, donde en ambos casos $i=c, h, f$ y $k=h, f, g$ con $i \neq k$ (Cuadro 2).

La Tabla 3 muestra que, para prácticamente todos los países, la persistencia agregada es un fenómeno más frecuente que la movilidad agregada excepto para China, único país en que la movilidad agregada es inferior al 50\%, tanto si es calculada a partir del número de clases tecnológicas como por el número de patentes. Las diferencias entre ambos fenómenos son apenas significativas para India y Corea del Sur, donde la persistencia afectó a más del 70\% de los campos técnicos concentrando también más del $70 \%$ de las patentes. En términos de campos técnicos, la persistencia se revela como un fenómeno asociado a la desespecialización y es más característico de países asiáticos cuyos pesos llegan a ser dos o tres veces más elevados que la persistencia en la especialización. En los países latinoamericanos, México mantiene diferencias muy reducidas y Brasil registra mayor propensión a persistir en la especialización. La persistencia de la especialización se concentra en competencias centrales para todos los países y con especial énfasis en el grupo de asiáticos, mientras que la persistencia de la desespecialización es un fenómeno específico de las competencias marginales. 

Tabla 3

Persistencia y Movilidad agregada entre periodos pre y post integración por país (\%)

\begin{tabular}{|c|c|c|c|c|c|c|c|c|c|c|}
\hline & & $\mathbf{A R}$ & BR & MX & $\mathrm{CN}$ & $\mathbf{K R}$ & HK & IN & SG & TW \\
\hline \multirow{12}{*}{ 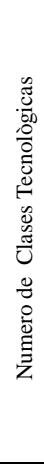 } & Persistencia agregada $\left(T_{j}\right)$ & 51,7 & 55,2 & 65,5 & 48,3 & 72,4 & 69,0 & 86,2 & 69,0 & 79,3 \\
\hline & Descomposición de la persistencia agregada & & & & & & & & & \\
\hline & Persistencia de la especialización $\left(P S_{j}\right)$ & 17,2 & 31,0 & 34,5 & 13,8 & 17,2 & 10,3 & 17,2 & 10,3 & 13,8 \\
\hline & En competencias Centrales & 10,3 & 24,1 & 27,6 & 13,8 & 13,8 & 10,3 & 17,2 & 6,9 & 13,8 \\
\hline & En competencias Nicho & 6,9 & 6,9 & 6,9 & 0,0 & 3,4 & 0,0 & 0,0 & 3,4 & 0,0 \\
\hline & Persistencia de la des-especialización $\left(P D_{j}\right)$ & 34,5 & 24,1 & 31,0 & 34,5 & 55,2 & 58,6 & 69,0 & 58,6 & 65,5 \\
\hline & En competencias de Fondo & 6,9 & 6,9 & 3,4 & 3,4 & 0,0 & 6,9 & 3,4 & 6,9 & 6,9 \\
\hline & En competencias Marginales & 27,6 & 17,2 & 27,6 & 31,0 & 55,2 & 51,7 & 65,5 & 51,7 & 58,6 \\
\hline & Movilidad agregada $\left(M_{j}^{T}\right)$ & 48,3 & 44,8 & 34,5 & 51,7 & 27,6 & 31,0 & 13,8 & 31,0 & 20,7 \\
\hline & $\begin{array}{l}\text { Descomposición de la movilidad agregada por componentes } \\
\text { Movilidad esperada }\left(M E_{i}^{T}\right)\end{array}$ & 3,4 & 3,4 & 3,4 & 3,4 & 3,4 & 0,0 & 0,0 & 10,3 & 6,9 \\
\hline & Movilidad no esperada (construcción) $\left(M N E C_{j}^{T}\right)$ & 13,8 & 27,6 & 20,7 & 6,9 & 3,4 & 24,1 & 10,3 & 6,9 & 3,4 \\
\hline & Movilidad no esperada (destrucción) $\left(M N E D_{j}^{T}\right)$ & 31,0 & 13,8 & 10,3 & 41,4 & 20,7 & 6,9 & 3,4 & 13,8 & 10,3 \\
\hline \multirow{12}{*}{ 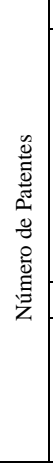 } & Persistencia agregada $\left(\boldsymbol{P}_{j}\right)$ & 54,9 & 59,3 & 62,1 & 41,5 & 79,3 & 75,0 & 83,2 & 66,4 & 69,3 \\
\hline & Descomposición de la persistencia agregada & & & & & & & & & \\
\hline & Persistencia de la especialización $\left(P S_{j}^{P}\right)$ & 38,5 & 47,4 & 51,7 & 25,0 & 65,2 & 44,0 & 56,9 & 38,8 & 39,2 \\
\hline & En competencias Centrales & 35,8 & 42,5 & 49,0 & 25,0 & 62,1 & 44,0 & 56,9 & 37,7 & 39,2 \\
\hline & En competencias Nicho & 2,6 & 4,9 & 2,6 & 0,0 & 3,2 & 0,0 & 0,0 & 1,1 & 0,0 \\
\hline & Persistencia de la des-especialización $\left(P D_{j}^{P}\right)$ & 16,4 & 11,9 & 10,5 & 16,5 & 14,1 & 31,0 & 26,3 & 27,6 & 30,2 \\
\hline & En competencias de Fondo & 9,1 & 7,3 & 3,7 & 3,6 & 0,0 & 10,3 & 9,2 & 9,1 & 7,7 \\
\hline & En competencias Marginales & 7,3 & 4,6 & 6,8 & 12,8 & 14,1 & 20,7 & 17,1 & 18,6 & 22,4 \\
\hline & Movilidad agregada (Mj) & 45,1 & 40,7 & 37,9 & 58,5 & 20,7 & 25,0 & 16,8 & 33,6 & 30,7 \\
\hline & $\begin{array}{l}\text { Descomposición de la movilidad agregada por componentes } \\
\text { Movilidad esperada }\left(M E_{j}^{P}\right)\end{array}$ & 6,3 & 3,8 & 5,6 & 33,8 & 3,7 & 0,0 & 0,0 & 17,6 & 21,6 \\
\hline & Movilidad no esperada (construcción) $\left(M N E C_{j}^{P}\right)$ & 15,6 & 26,5 & 25,8 & 8,0 & 4,7 & 12,0 & 14,8 & 5,5 & 3,0 \\
\hline & Movilidad no esperada (destrucción) $\left(M N E D_{j}^{P}\right)$ & 23,3 & 10,4 & 6,5 & 16,7 & 12,4 & 13,0 & 2,0 & 10,4 & 6,0 \\
\hline
\end{tabular}

(AR), Argentina; (BR), Brasil; (MX), México; (CN), China; (KR) Corea del Sur; (HK), Hong-Kong; (IN), India; (SG), Singapur; (TW), Taiwán.

Fuente: Elaboración propia. 

En términos de patentes, como esperado dada la forma en que se define este indicador, la persistencia en la especialización es claramente superior a la persistencia en la des-especialización ${ }^{3}$, siendo fuertemente concentrada en competencias centrales en el primer caso y en competencias marginales en el segundo para la mayor parte de los países. La teoría de la acumulación tecnológica y la existencia de capacidad de absorción previa explican estos fenómenos. La creación de competencias y capacidades en el pasado permiten el mantenimiento de fortalezas tecnológicas en el futuro, así como la inexistencia de esfuerzos previos limita la creación de capacidades y capacitaciones futuras.

En cuanto al comportamiento de la movilidad, algunos efectos relevantes pueden ser observados. En primer lugar, para todos los países contemplados, cuando la movilidad se mide por número de campos técnicos, ésta se caracteriza más por ser 'no esperada' que 'esperada'. Sólo cuando la movilidad es medida en términos del número de patentes, la movilidad esperada es superior a la no esperada en los casos de China, Singapur y Taiwán (Tabla 3). Salvo en estos países, la movilidad esperada adquiere un cierto carácter residual. De esta forma, la teoría de la acumulación tecnológica, si bien conseguía explicar los fenómenos observados para la persistencia, se muestra insuficiente para explicar la naturaleza del fenómeno relativo a movilidad.

En el grupo de países contemplados se revelan dos patrones sobre movilidad no esperada. Por un lado, los tres latinoamericanos (Brasil, Argentina y México) junto con India y Hong-Kong forman un grupo donde la movilidad se caracteriza más por la creación de nuevas competencias que por la destrucción de las viejas. En el caso latinoamericano, donde además la movilidad es un fenómeno casi tan importante como la persistencia, la falta de madurez tecnológica puede explicar una menor dependencia de situaciones de lock-in, lo que permitiría la movilidad hacia la creación de competencias en tecnologías donde no necesariamente había capacidad previa. El caso de India y Hong-Kong es diferente. Estos países revelaron elevados índices de persistencia, lo que lleva a pensar que hay un cierto grado de dependencia del pasado. La creación no esperada de nuevas competencias puede deberse a un efecto inducido de las políticas tecnológicas o decurrente de los vínculos y spillovers entre tecnologías. El segundo patrón de movilidad, formado por China, Corea del Sur, Singapur y Taiwán, se caracteriza más por la destrucción de competencias pasadas. Los casos de China y Corea se explican en buena medida por una reconducción de sus esfuerzos tecnológicos hacia la concentración. China registró perdida de competencias en el 41,4\% de los campos técnicos, los cuales concentraron apenas el 16,7\% de las patentes en el

(3) En términos del número de patentes es esperado que la especialización tenga un mayor peso que la des-especialización, dado que de acuerdo con la VTR, los campos donde hay especialización son aquellos donde un país patenta más en términos relativos. 
periodo post-integración. Corea del Sur perdió competencias en el 20,7\% de sus campos técnicos, absorbiendo apenas el $12,4 \%$ de sus patentes en el periodo postintegración.

El análisis de la persistencia y movilidad en función del dinamismo de la OT por campo técnico permite cualificar mejor la idea acerca de la existencia de una división internacional del 'trabajo en actividad innovadora' que relega a los países latinoamericanos al estancamiento (especialización en tecnologías estancadas), mientras que otorga a los asiáticos especializaciones en tecnologías dinámicas. En relación con la persistencia, la Tabla 4 permite observar que las propensiones sectoriales a mantener especializaciones (y des-especializaciones) iniciales son siempre superiores en América Latina que en Asia. Sin embargo, los países asiáticos consiguen concentrar el 42,7\% de sus patentes en las tecnologías dinámicas en donde están especializados, mientras que en América Latina ese porcentaje es de sólo del 21,3\%. Por otro lado, los países latinoamericanos concentran el $25,4 \%$ de sus patentes en especializaciones en campos técnicos con OT estancada entre periodos. Los anexos 1 y 2 permiten observar que los países asiáticos tienden a especializarse en tecnologías vinculadas al paradigma de las telecomunicaciones, mientras que los latinoamericanos tienden a especializarse más en tecnologías relacionadas con la explotación de recursos naturales. En los países asiáticos, los campos técnicos dinámicos que persistieron como competencias centrales fueron las tecnologías de la información, audiovisuales y telecomunicaciones, y aparatos electrónicos e ingeniería eléctrica. También se mantuvieron como competencias centrales las tecnologías farmacéutica y cosmética en China e India; la biotecnología en India; las de transporte en Taiwán y las relacionadas con química del petróleo en Corea del Sur (Anexo 1). Mientras, los países latinoamericanos mantuvieron sus competencias centrales y nicho en los campos técnicos dinámicos relativos a tecnologías médicas y química del petróleo. Brasil mantuvo su competencia central en motores y bombas; Brasil y México en biotecnología; y Argentina y México en farmacéutica y cosmética (Anexo 1).

Entre las tecnologías de OT estancada, prácticamente todos los países contemplados (excepto India y Singapur) mantuvieron especializaciones centrales en bienes de consumo. En el resto, la distribución es aleatoria y no se observa un patrón claro de especialización por tipo de tecnología (Anexo 1).

En lo que se refiere a movilidad, cabe destacar las siguientes observaciones. En primer lugar, mientras los países latinoamericanos no registraron movilidad alguna hacia sectores dinámicos relacionada con competencias previas, los países asiáticos deben el 10,8\% de sus patentes en el periodo post-integración a movimientos en esta dirección. Por el contrario, la movilidad en los países latinoamericanos se caracteriza por ser no esperada, construyendo nuevas competencias tanto en tecnologías dinámicas $(12,2 \%$ de sus patentes en el periodo 
post-integración) como en tecnologías de OT estancada (12,0\%). El Anexo 2 permite observar que la movilidad 'esperada' sólo tuvo lugar en la creación de competencias centrales. En el grupo de países asiáticos, la movilidad esperada se dirige hacia tecnologías de OT dinámica de forma muy diversa; Corea del Sur, Singapur y Taiwán hacia semiconductores; China y Taiwán hacia tecnologías audiovisuales y telecomunicaciones; y Singapur hacia biotecnología. Sin embargo, en el caso latinoamericano se dirige hacia tecnologías de OT estancada (Argentina en Handling, Brasil en química molecular y polímeros y México en agricultura y alimentos) (Anexo 2). Este resultado es coherente con el signo negativo del efecto adaptación de los países latinoamericanos en el análisis shift-share y con el signo positivo observado para los asiáticos.

En lo que se refiere a la destrucción de competencias, no se observan diferencias significativas entre ambos grupos de países, tanto en tecnologías de OT dinámica como estancada (Tabla 4).

Tabla 4

Persistencia y Movilidad de acuerdo con el dinamismo de la oportunidad tecnológica.

Comparación América Latina-Asia. (\%)

\begin{tabular}{|c|c|c|c|c|c|}
\hline & & \multicolumn{2}{|c|}{ Campos técnicos } & \multicolumn{2}{|c|}{ Patentes } \\
\hline & & $\begin{array}{l}\text { América } \\
\text { Latina }\end{array}$ & Asia & $\begin{array}{l}\text { América } \\
\text { Latina }\end{array}$ & Asia \\
\hline \multirow{5}{*}{$\begin{array}{c}\text { Tecnologias de } \\
\text { Oportunidad } \\
\text { Tecnologica } \\
\text { Dinámica }\end{array}$} & $\begin{array}{l}\text { Persistencia de la } \\
\text { especialización }\end{array}$ & 12,6 & 8,0 & 21,3 & 42,7 \\
\hline & $\begin{array}{l}\text { Persistencia de la des- } \\
\text { especialización }\end{array}$ & 11,5 & 17,2 & 8,6 & 7,1 \\
\hline & Movilidad esperada & 0,0 & 3,5 & 0,0 & 10,8 \\
\hline & $\begin{array}{l}\text { Movilidad no esperada } \\
\text { (construcción) }\end{array}$ & 6,9 & 3,5 & 12,2 & 2,7 \\
\hline & $\begin{array}{l}\text { Movilidad no esperada } \\
\text { (destrucción) }\end{array}$ & 6,9 & 5,7 & 4,5 & 5,7 \\
\hline \multirow{6}{*}{$\begin{array}{c}\text { Tecnologias de } \\
\text { Oportunidad } \\
\text { Tecnologica } \\
\text { Estancada }\end{array}$} & $\begin{array}{l}\text { Persistencia de la } \\
\text { especialización }\end{array}$ & 14,9 & 5,7 & 25,4 & 10,7 \\
\hline & $\begin{array}{l}\text { Persistencia de la des- } \\
\text { especialización }\end{array}$ & 18,4 & 39,7 & 3,8 & 11,0 \\
\hline & Movilidad esperada & 3,5 & 0,6 & 4,7 & 0,2 \\
\hline & $\begin{array}{l}\text { Movilidad no esperada } \\
\text { (construcción) }\end{array}$ & 13,8 & 5,8 & 12,0 & 3,3 \\
\hline & $\begin{array}{l}\text { Movilidad no esperada } \\
\text { (destrucción) }\end{array}$ & 11,5 & 10,3 & 7,5 & 5,8 \\
\hline & Total & $87 *$ & $174 *$ & 2609 & 43175 \\
\hline
\end{tabular}

(*): 29 clases tecnológicas por 3 países latinoamericanos (87) y por 6 países asiáticos (174).

Fuente: Elaboración propia. 
En resumen, el análisis de la movilidad y persistencia desde el punto de vista de las competencias tecnológicas nacionales parece corroborar las hipótesis sobre el importante papel de la acumulación tecnológica para explicar la persistencia de la especialización en tecnologías que representan fortalezas tecnológicas para el país, la persistencia de la des-especialización en los campos técnicos que son sus mayores debilidades y la construcción de nuevas competencias a partir de competencias previas para el caso de los países asiáticos. Sin embargo, la teoría de la acumulación tecnológica poco ayuda a explicar la naturaleza de la movilidad en el caso de los países latinoamericanos donde una parte significativa de sus especializaciones se dirigió hacia sectores donde no existían competencias previas.

Por otro lado, la creación de competencias tiene lugar independientemente del carácter dinámico o estancado de la oportunidad tecnológica asociada a cada campo técnico, lo que permite deducir que la movilidad no se caracteriza por el aprovechamiento de las oportunidades que ofrece el dinamismo tecnológico a lo largo del tiempo. Es también por esta razón que la especialización tecnológica inicial no parece representar un handicap a la movilidad hacia otros campos técnicos (dinámicos o estancados). La restricción a la movilidad parece estar más asociada a las especificidades de la estructura productiva de cada país y a las posibilidades que ofrecen las tecnologías vinculadas con ella.

\section{Conclusiones}

Este trabajo tenía como objetivo observar comparativamente los cambios registrados en las estructuras tecnológicas entre dos grupos de países (uno latinoamericano y otro asiático) y entre dos situaciones referidas a lo que serían momentos de pre y post integración de sus mercados de bienes, servicios y capitales. El propósito de tal comparación era determinar si hubo captura (elevado dinamismo tecnológico) y si 'correctas' o 'incorrectas' especializaciones iniciales habrían representado una restricción a este proceso. En caso de confirmarse la captura, el siguiente objetivo sería saber si la captura vino acompañada de convergencia y de cambio en las estructuras tecnológicas nacionales hacia una mayor especialización o diversificación. Una vez comprobado el cambio estructural, un análisis de la evolución de las competencias nacionales permitiría identificar si la evolución de las ETN sigue la lógica de la acumulación tecnológica o si factores relacionados con estímulos inducidos o con cambio estructural deben ser más relevantes como elementos que explican la evolución de los patrones de ETN en periodos de integración económica.

El artículo permite confirmar que hubo un elevado dinamismo tecnológico en todos los países excepto en Hong-Kong, mas éste era un país que ya partía de una situación de elevado nivel de integración internacional en el periodo 
considerado de pre-apertura. El dinamismo tecnológico sucedió independientemente del posicionamiento inicial dado el carácter marginal del efecto estructural. La captura no se tradujo en convergencia para todos los países. En cuanto una parte de ellos redujo sus distancias con el mundo (México, India, Singapur y Taiwán), otros las aumentaron (Argentina, Brasil, China y Corea del Sur). La divergencia viene explicada, sobre todo, por el aumento de la especialización y concentración de estos países en un menor número de campos técnicos, siendo especialmente relevante en China y Corea del Sur.

El estudio confirma que hubo cambio de las estructuras tecnológicas entre los periodos establecidos como de pre y post integración. La persistencia es un fenómeno más asociado a la des-especialización que a la especialización. Ésta se concentra principalmente en competencias marginales y es más característica de los países asiáticos que de los latinoamericanos. La movilidad, por su parte, parece responder a un proceso de construcción de competencias sobre esfuerzos previos en el caso asiático, pero no en el latinoamericano, donde una parte significativa de las competencias centrales y nicho en el periodo post-apertura, y tanto en tecnologías de OT dinámica como estancada, eran competencias marginales en el periodo pre-apertura. Esto lleva a suponer que factores no relacionados con el proceso de acumulación tecnológica tuvieron un importante papel a lo largo del proceso de integración de mercados en los países latinoamericanos contemplados. Estimar la medida en que el cambio de estructuras tecnológicas se debe a cambios de las estructuras productivas es objeto de futuros trabajos.

Finalmente, el artículo permite verificar una mayor propensión de los países latinoamericanos a permanecer y moverse hacia tecnologías con OT estancada entre periodos, mientras que los asiáticos mostraron una mayor propensión a concentrar sus esfuerzos tecnológicos y a moverse hacia tecnologías de OT dinámica. Sin embargo, no hay evidencia de que este tipo de movimiento haya representado un handicap al dinamismo tecnológico registrado por los países latinoamericanos.

\section{Bibliografía}

ALCORTA, L.; PERES, W. Innovation systems and technological specialisation in Latin America and the Caribbean. Research Policy, 26, p. 857-881, 1998.

ARCHIBUGI, D.; COCO, A. Measuring technological capabilities at the country level: a survey and a menu for choice. Research Policy, 34, p. 175-194, 2005.

; PIANTA, M. The technological specialization of advanced countries. Dordrecht: Kluwer Academic, 1992.

Aggregate convergence and sectoral specialisation in innovation. Journal of Evolutionary Economics, 4, p. 17-33, 1994. 
BRUSONI, S.; GEUNA, A. An international comparison of sectoral knowledge bases: persistence and integration in the pharmaceutical industry. Research Policy, 32, p. 18971912, 2003.

CAMERONA, G.; PROUDMANB, J.; REDDING, S. Technological convergence, R\&D, trade and productivity growth. European Economic Review, 49, p. 775-807, 2005.

COHEN, W.M. Empirical studies of innovative activity. In: STONEMAN, P. (Ed.). Handbook of the economics of innovation and technological change. Oxford: Blackwell Publishers Ltd., 1995. p. 183-264.

; LEVINTHAL, D. A. Absorptive capacity: a new perspective on learning and innovation. Administrative Science Quarterly, 35, p. 128-152, 1990.

DOSI, G. Sources, procedures and microeconomics effects of innovation. Journal of Economic Literature, 26, p. 1120-1171, 1988.

GRUPP, H.; SCHOMACH, U. Patent statistics in the age of globalisation: new legal procedures, new analytical methods, new economic interpretation. Research Policy, 28, p. 377-396, 1999.

GWARTNEY, J.; HALL, J.; LAWSON, R. Economic freedom of the world: 2010 Annual Report. Vancouver, BC: The Fraser Institute, 2010. Datos extraídos del web-site: www.freetheworld.com.

HUANG, H. T.; MIOZZO, M. Patterns of technological specialisation in Latin-American and East Asian countries: an analysis of patents and trade flows. Economic Innovation and New Technologies, v. 13, n. 7, p. 615-653, 2004.

IMBS, J.; WACZIARG, R. Stages of diversification. American Economic Review, v. 93, n. 1, p. 63-86, 2003.

JUNGMITTAG, A. Innovations, technological specialisation and economic growth in the EU. International Economics and Economic Policy, 1, p. 247-273, 2004.

LALL, S. The technological structure and performance of developing country manufactured exports, 1985-98. Oxford Development Studies, v. 28, n. 3, p. 338-363, 2000.

LAURSEN, K. The impact of technological opportunity on the dynamics of trade performance. Structural Change and Economic Dynamics, 10, p. 341-357, 1999.

LE BAS, C.; SIERRA, C. Location versus home country advantages. In: R\&D activities: some further results on multinationals' locational strategies. Research Policy, 31, p. 589609, 2002.

MALERBA, F.; ORSENIGO, L.; PERETTO, P. Persistence of innovative activities, sectoral patterns of innovation and international technological specialization. International Journal of Industrial Organization, 15, p. 801-826, 1997.

MALERBA, F.; MONTOBBIO, F. Exploring factors affecting international technological specialization. Journal of Evolutionary Economics, 13, p. 411-434, 2003. 
MANCUSI, M. L. International technological specialization in industrial countries: patterns and dynamics. Weltwirtschaftliches Archiv, v. 137, n. 4, p. 593-621, 2001.

Geographical concentration and the dynamics of countries specialization. Economics of Innovation and New Technologies, v. 12, n. 3, p. 269-291, 2003.

MELICIANI, V. The impact of technological specialisation on national performance in a balance-of-payments-constrained growth model. Structural Change and Economic Dynamics, 13, p. 101-118, 2002.

MIOZZO, M. Sectoral specialisation in East Asia and Latin America compared. Brazilian Journal of Political Economy, v. 22, n. 4 (88), p. 48-68, 2002.

MONTOBBIO, F.; RAMPA, F. The impact of technology and structural change on export performance in nine developing countries. World Development, v. 33, n. 4, p. 527-547, 2005.

NELSON, R.; WINTER, S. In search of a useful theory of innovation. Research Policy, v. 6, n. 1, p. 36-76, 1977.

PATEL, P.; PAVITT, K. The technological competences in the world's largest firms: complex and path dependent, but not much variety. Research Policy, 26, p. 141-156, 1997.

PIANTA, M.; MELICIANI, V. Technological specialization and economic performance in OECD countries. Technology Analysis \& Strategic Management, v. 8, n. 2, p. 157-174, 1996.

STOLPE, M. Technology and the dynamics of specialization in open economies. Tubingen Mohr, 1995.

UNCTAD. Handbook of statistics. United Nations, 2009.

URRACA-RUIZ, A. The 'technological' dimension of structural change under market integration. Structural Change and Economic Dynamics, 27, p. 1-18, 2013.

VERTOVA, G. National technological specialisation and the highest technological opportunities historically. Technovation, 21, p. 605-612, 2001.

Van ZEEBROECK, N.; Van POTTELSBERGHE de la POTTERIE, B.; HAN, W. Issues in measuring the degree of technological specialisation with patent data. Scientometrics, v. 66, n. 3, p. 481-492, 2006 



\section{Anexo 1}

Persistencia de las competencias tecnológicas por campo técnico entre periodos pre y post integración

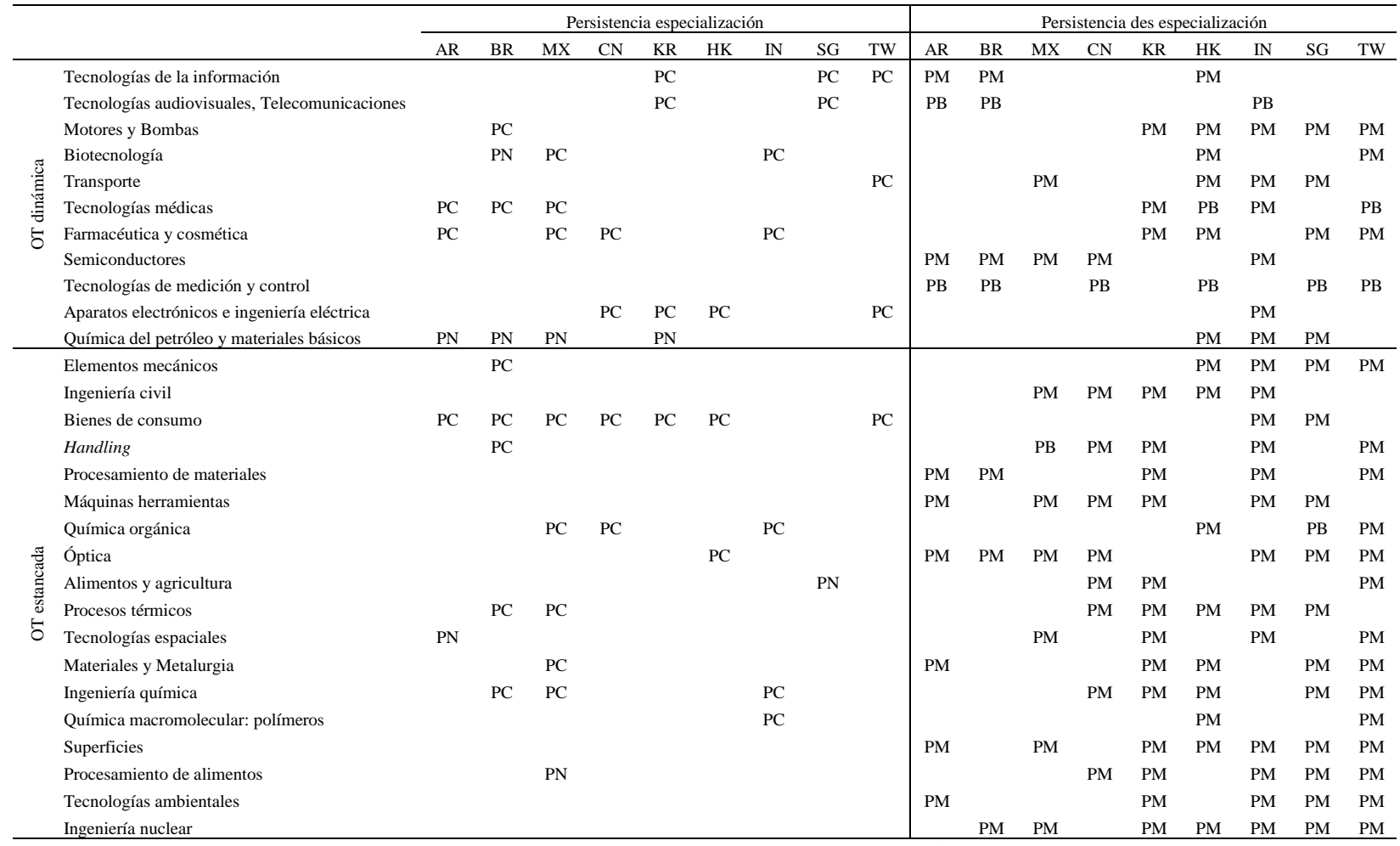

Nota: Entre periodos pre y post integración cada tecnología; (PC), persistió como competencia central; (PN) persistió como competencia Nicho; (PB) persistió como competencia 'de Fondo'; (PM) persistió como competencia Marginal. (AR), Argentina; (BR), Brasil; (MX), México; (CN), China; (KR) Corea del Sur; (HK), Hong-Kong; (IN), India; (SG), Singapur; (TW), Taiwán.

Fuente: Elaboración propia. 



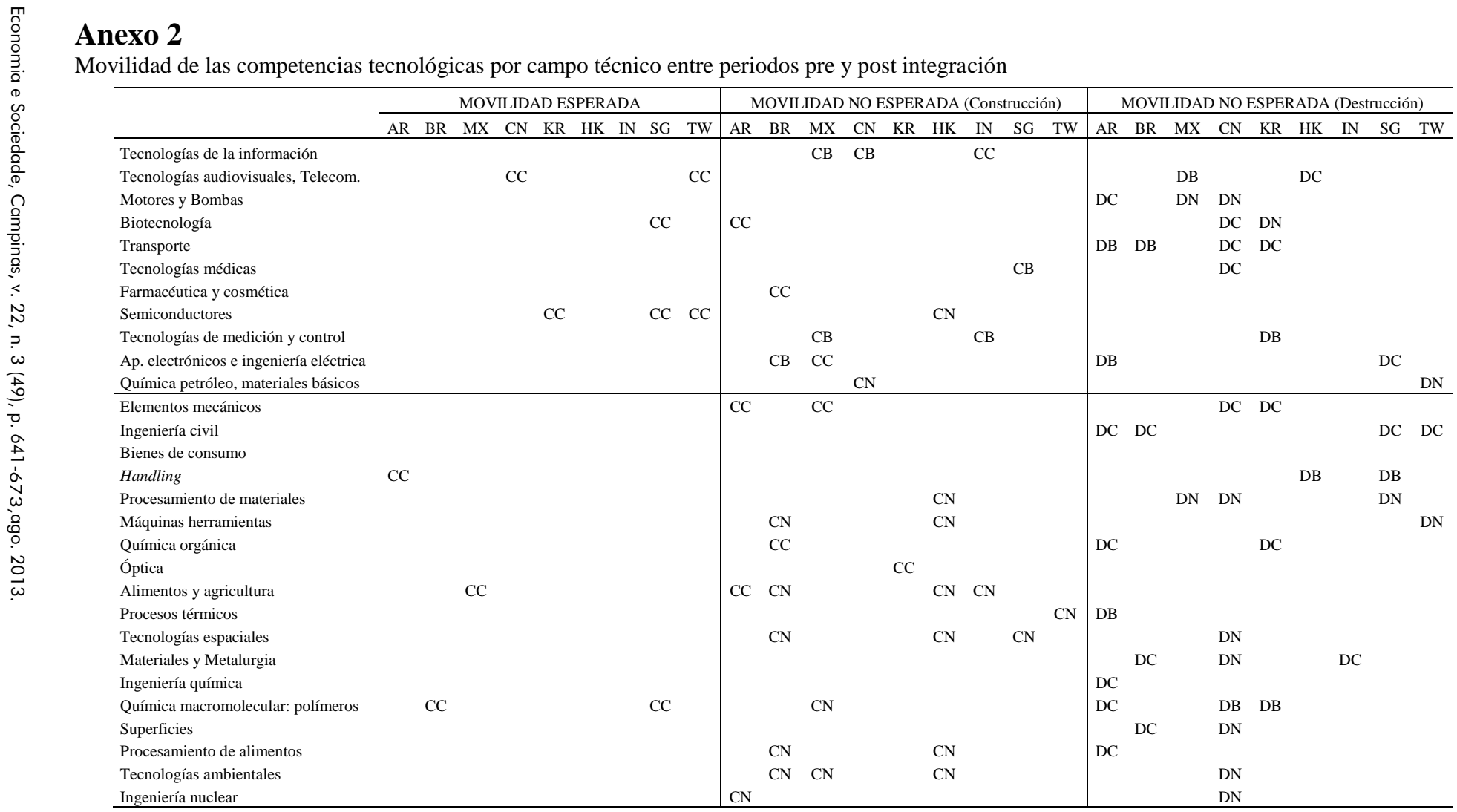

Nota: Entre periodos pre y post integración cada tecnología; (CC) pasó a ser caracterizada como competencia Central; (CN) pasó a ser caracterizada como competencia Nicho; (CB) pasó a ser caracterizada como competencia de fondo; (DC), se descaracterizó como competencia Central; (DN), se descaracterizó como competencia Nicho; (DB) se descaracterizó como competencia de Fondo. (AR), Argentina; (BR), Brasil; (MX), México; (CN), China; (KR) Corea del Sur; (HK), Hong-Kong; (IN), India; (SG), Singapur; (TW), Taiwan.

Fuente: Elaboración propia. 